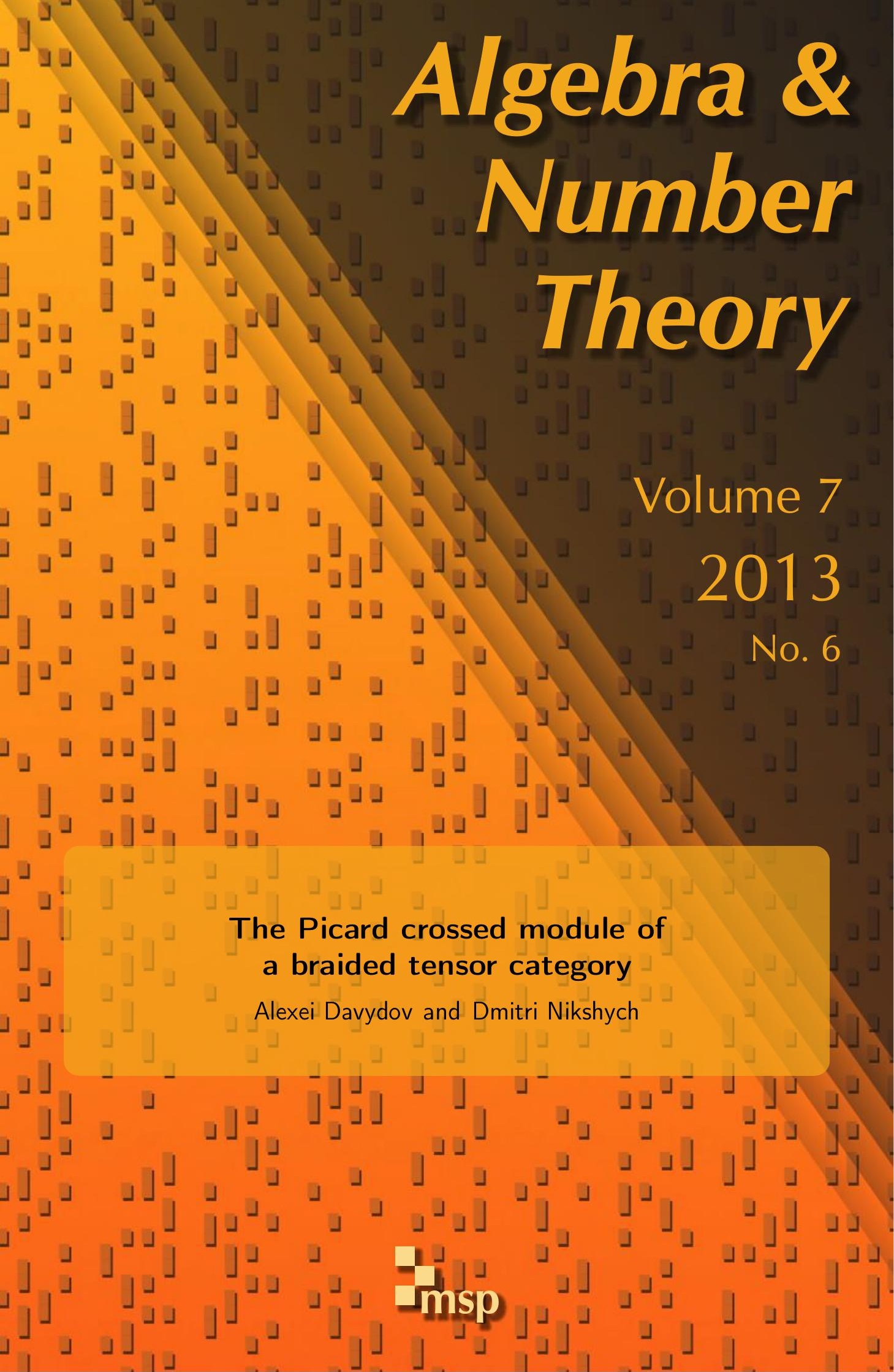




\section{The Picard crossed module of a braided tensor category}

Alexei Davydov and Dmitri Nikshych

For a finite braided tensor category $\mathscr{b}$ we introduce its Picard crossed module $\mathfrak{P}(\mathscr{C})$ consisting of the group of invertible $\mathscr{C}$-module categories and the group of braided tensor autoequivalences of $\mathscr{C}$. We describe $\mathfrak{P}(\mathscr{C})$ in terms of braided autoequivalences of the Drinfeld center of $\mathscr{b}$. As an illustration, we compute the Picard crossed module of a braided pointed fusion category.

\section{Introduction}

Tensor categories can be thought of as categorical analogues of associative algebras. One can adapt standard notions and constructions of the classical theory of associative algebras to tensor categories. Analogues of (bi-)modules over algebras are (bi-@)module categories over tensor categories [Quillen 1973; Janelidze and Kelly 2001; Ostrik 2003b].

Given an algebra $C$ the isomorphism classes of invertible $C$-bimodules form a group $\operatorname{BrPic}(C)$ called the Brauer-Picard group of $C$. There is a well-known homomorphism

$$
\phi: \operatorname{BrPic}(\mathscr{C}) \rightarrow \operatorname{Aut}(Z(C)),
$$

where $Z(C)$ denotes the center of $C$, constructed as follows. Given an invertible $C$-bimodule $M$ and $z \in Z(C)$, the element $\phi(M)(z) \in Z(C)$ is defined by the condition that the endomorphism of $M$ given by the left multiplication by $\phi(M)(z)$ equals that given by the right multiplication by $z$.

There is an analogue of the homomorphism (1) for tensor categories. Given a finite tensor category $\mathscr{C}$ one defines its Brauer-Picard group BrPic $(\mathscr{C})$ of equivalence classes of invertible $\mathscr{C}$-bimodule categories (see [Etingof et al. 2010]) and a homomorphism

$$
\Phi: \operatorname{BrPic}(\mathscr{C}) \rightarrow \operatorname{Aut}^{\mathrm{br}}(\mathscr{L}(\mathscr{C}))
$$

Nikshych's work was partially supported by the NSF grant DMS-0800545.

MSC2010: primary 18D10; secondary 16W30.

Keywords: braided tensor category, Drinfeld center, braided autoequivalence, invertible module category. 
where $\mathscr{L}(\mathscr{C})$ is the Drinfeld center of $\mathscr{C}$ and $\operatorname{Aut}^{\text {br }}(\mathscr{L}(\mathscr{C}))$ is the group of braided autoequivalences of $\mathscr{L}(\mathscr{C})$.

It was shown in [Etingof et al. 2010] that (2) is an isomorphism when $\mathscr{C}$ is a fusion category.

Braided tensor categories are analogues of commutative algebras. Similarly to the classical case, module categories over a braided tensor category $\mathscr{C}$ can be regarded as bimodule categories. In this case the group $\operatorname{BrPic}(\mathscr{C})$ contains a subgroup $\operatorname{Pic}(\mathscr{C})$, called the Picard group of $\mathscr{C}$, consisting of invertible $\mathscr{b}$-module categories [Etingof et al. 2010]. One defines a homomorphism

$$
\partial: \operatorname{Pic}(\mathscr{C}) \rightarrow \operatorname{Aut}^{\mathrm{br}}(\mathscr{C})
$$

in a way parallel to (2). The classical analogue of (3) for commutative algebras is trivial, but in general $\partial$ is far from being trivial. It was shown in [Etingof et al. $2010]$ that it is an isomorphism for every nondegenerate braided fusion category $\mathscr{C}$.

Groups Pic $(\mathscr{C})$ and $\mathrm{Aut}^{\mathrm{br}}(\mathscr{C})$ play important roles in the theory of braided tensor categories. In particular, they are used in the classification of group extensions of fusion categories [Etingof et al. 2010]. They also appear as parts of an important invariant of $\mathscr{C}$ called the core, studied in [Drinfeld et al. 2010]. We thus hope that our description of the algebraic structure formed by these groups will shed more light on these constructions.

The starting point of this paper is a conjecture of V. Drinfeld that for a braided tensor category $\mathscr{C}$ the pair $\mathfrak{P}(\mathscr{C})=\left(\operatorname{Pic}(\mathscr{C})\right.$, $\left.\operatorname{Aut}^{\mathrm{br}}(\mathscr{C})\right)$ along with the homomorphism (3) and the natural action of $\operatorname{Aut}^{\text {br }}(\mathscr{C})$ on $\operatorname{Pic}(\mathscr{C})$ is a crossed module, called the Picard crossed module of $\mathscr{C}$. See Section 3D for the definition of a crossed module and [Joyal and Street 1993; Drinfeld et al. 2010, Appendix E.5.3] for an interpretation of crossed modules in terms of monoidal categories. We prove this conjecture in Theorem 3.10.

For a finite tensor category $\mathscr{C}$ we define its Brauer-Picard group $\operatorname{BrPic}(\mathscr{C})$ as the group of equivalence classes of invertible exact $\mathscr{C}$-bimodule categories. We prove in Theorem 4.1 that the canonical homomorphism (2) is an isomorphism. This extends the corresponding result for fusion categories proved in [Etingof et al. 2010].

Next, for a braided finite tensor category $\mathscr{C}$ we show in Theorem 4.3 that the image of $\operatorname{Pic}(\mathscr{C}) \subset \operatorname{BrPic}(\mathscr{C})$ under the isomorphism (2) is the subgroup of braided autoequivalences of $\mathscr{L}(\mathscr{C})$ trivializable on $\mathscr{C}$.

Finally, we explicitly compute the Picard crossed module of a pointed braided fusion category in Section 5. It turns out that the Picard groups of pointed braided fusion categories interpolate between the orthogonal groups of quadratic forms and the exterior squares of finite abelian groups. 
The paper is organized as follows.

Section 2 contains basic facts about finite tensor categories and module categories over them. Here we also define the Brauer-Picard group of a finite tensor category and the Picard group of a finite braided tensor category. (They were previously defined in [Etingof et al. 2010] in the setting of fusion categories.)

In Section 3 we introduce the Picard crossed module of a braided tensor category.

In Section 4 we prove our Main Theorems 4.1 and 4.3 and describe the Picard crossed module of a braided tensor category in terms of braided autoequivalences of its center.

Section 5 is devoted to the computation of the Picard crossed module of a pointed braided fusion category and its invariants.

\section{Preliminaries}

2A. General conventions. We work over an algebraically closed field $k$. Recall that a $k$-linear abelian category $\mathscr{C}$ is finite if

(i) $\mathscr{C}$ has finite dimensional spaces of morphisms;

(ii) every object of $\mathscr{C}$ has finite length;

(iii) $\mathscr{C}$ has enough projectives, that is, every simple object of $\mathscr{C}$ has a projective cover; and

(iv) there are finitely many isomorphism classes of simple objects in $\mathscr{b}$.

All abelian categories considered in this paper will be finite. Any such category is equivalent to the category $\operatorname{Rep}(A)$ of finite dimensional representations of a finite dimensional $k$-algebra $A$. All functors between such categories will be additive and $k$-linear. We use the symbol $\simeq$ for equivalence between categories and the symbol $\cong$ for isomorphisms between objects.

In this paper we freely use basic results of the theory of finite tensor categories and module categories over them [Bakalov and Kirillov 2001; Etingof and Ostrik 2004; Ostrik 2003b] and the theory of braided categories [Joyal and Street 1993; Drinfeld et al. 2010].

2B. Tensor categories. By a tensor category we mean a finite rigid tensor category $\mathscr{A}$ whose unit object 1 is simple [Etingof and Ostrik 2004]. A semisimple tensor category is called a fusion category.

Let $\mathscr{A}$ be a tensor category with the associativity constraint

$$
a_{X, Y, Z}:(X \otimes Y) \otimes Z \stackrel{\simeq}{\rightarrow} X \otimes(Y \otimes Z) .
$$

The tensor category with the opposite tensor product $X \otimes{ }^{\mathrm{op}} Y:=Y \otimes X$ and the 
accordingly adjusted associativity constraint $a^{\text {op }}$

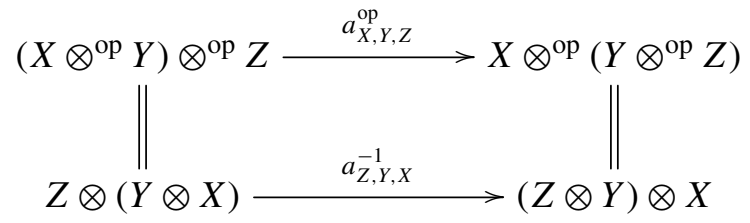

will be called the category opposite to $\mathscr{A}$ and will be denoted $\mathscr{A}^{\mathrm{op}}$.

Let $\mathscr{A}$ and $\mathscr{B}$ be tensor categories. Their Deligne tensor product [Deligne 2002] will be denoted by $\mathscr{A} \otimes \mathscr{B}$.

Definition 2.1. Let $\mathscr{A}$ be a tensor category and let $\mathscr{B} \subset \mathscr{A}$ be a tensor subcategory. A tensor autoequivalence $\alpha$ of $\mathscr{A}$ is called trivializable on $\mathscr{B}$ if the restriction $\left.\alpha\right|_{\mathscr{B}}$ is isomorphic to $\mathrm{id}_{\mathscr{B}}$ as a tensor functor.

We will denote by $\operatorname{Aut}(\mathscr{A})($ respectively, $\operatorname{Aut}(\mathscr{A}, \mathscr{B}))$ the group of isomorphism classes of tensor autoequivalences of $\mathscr{A}$ (respectively, tensor autoequivalences of $\mathscr{A}$ trivializable on $\mathscr{B})$.

2C. Braided tensor categories. Recall that a braided tensor category $\mathscr{b}$ is a finite tensor category equipped with a natural isomorphism

$$
c_{X, Y}: X \otimes Y \stackrel{\sim}{\rightarrow} Y \otimes X
$$

satisfying the hexagon axioms [Joyal and Street 1993]. The braiding of $\mathscr{C}$ gives rise to a tensor equivalence between $\mathscr{C}$ and $\mathscr{C}^{\mathrm{op}}$.

An important example of a braided tensor category is the center $\mathscr{L}(\mathscr{A})$ of a finite tensor category $\mathscr{A}$. It is defined as the category whose objects are pairs $(Z, \gamma)$, where $X$ is an object of $\mathscr{A}$ and $\gamma$ is a natural family of isomorphisms

$$
\gamma_{X}: X \otimes Z \stackrel{\simeq}{\rightarrow} Z \otimes X, \quad X \in \mathscr{A},
$$

called half-braidings, satisfying compatibility conditions. The center is a finite braided tensor category with the braiding given by

$$
\delta_{Z}:(Z, \gamma) \otimes(Y, \delta) \stackrel{\simeq}{\rightarrow}(Y, \delta) \otimes(Z, \gamma) .
$$

Let $\mathscr{C}^{\text {rev }}$ denote the tensor category $\mathscr{C}$ equipped with the reversed braiding

$$
\tilde{c}_{X, Y}=c_{Y, X}^{-1} .
$$

For a braided tensor category $\mathscr{C}$ there are canonical embeddings $\mathscr{C} \hookrightarrow \mathscr{L}(\mathscr{b})$ and $\mathscr{C}^{\text {rev }} \hookrightarrow \mathscr{L}(\mathscr{C})$ given by

$$
X \mapsto\left(X, c_{-, X}\right) \quad \text { and } \quad X \mapsto\left(X, \tilde{c}_{-, X}\right) .
$$


For a braided tensor category $\mathscr{b}$ the embeddings (4) combine into a single braided tensor functor

$$
\mathscr{C} \nabla \mathscr{C}^{\text {rev }} \rightarrow \mathscr{L}(\mathscr{C})
$$

A braided tensor category $\mathscr{b}$ is called factorizable if the functor (5) is an equivalence.

We will denote by $\mathrm{Aut}^{\mathrm{br}}(\mathscr{C})$ the group of isomorphism classes of braided tensor autoequivalences of a braided tensor category $\mathscr{C}$.

Recall that a tensor category is called pointed if its every simple object is invertible.

Example 2.2. Let $\mathscr{b}$ be a pointed braided fusion category. Then isomorphism classes of simple objects of $\mathscr{C}$ form a finite abelian group $A$.

The associativity constraint of $\mathscr{C}$ determines a 3-cocycle $\omega: A \times A \times A \rightarrow k^{\times}$. The braiding determines a function

$$
c: A \times A \rightarrow k^{\times}
$$

satisfying the following identities coming from the hexagon axioms of the braided tensor category:

$$
\begin{aligned}
& c(x, y+z) c(x, y)^{-1} c(x, z)^{-1}=\omega(x, y, z) \omega(y, x, z)^{-1} \omega(y, z, x), \\
& c(x+y, z) c(x, z)^{-1} c(y, z)^{-1}=\omega(x, y, z)^{-1} \omega(x, z, y) \omega(z, x, y)^{-1},
\end{aligned}
$$

for all $x, y, z \in A$. Following [Eilenberg and Mac Lane 1953; 1954], we denote by $Z_{a b}^{3}\left(A, k^{\times}\right)$the set of pairs $(\omega, c)$, where $\omega$ is a 3-cocycle on $A$ and $c$ is a function satisfying (7) and (8). Note that $Z_{a b}^{3}\left(A, k^{\times}\right)$is a group with respect to pointwise multiplication.

Thus, every pointed braided fusion category determines an element of $Z_{a b}^{3}\left(A, k^{\times}\right)$. Conversely, given $(\omega, c) \in Z_{a b}^{3}\left(A, k^{\times}\right)$one defines a braided category structure on the fusion category $\operatorname{Vec}_{A}$ of finite dimensional $A$-graded vector spaces using $\omega$ for the associativity constraint and $c$ for braiding.

Let $\mathscr{C}$ and $\mathscr{C}^{\prime}$ be pointed braided fusion categories corresponding to $(\omega, c) \in$ $Z_{a b}^{3}\left(A, k^{\times}\right)$and $\left(\omega^{\prime}, c^{\prime}\right) \in Z_{a b}^{3}\left(A^{\prime}, k^{\times}\right)$, respectively. A tensor functor $F: \mathscr{C} \rightarrow \mathscr{C}^{\prime}$ gives rise to a group homomorphism $f: A \rightarrow A^{\prime}$. The tensor structure of $F$ gives rise to a map $\phi: A \times A \rightarrow k^{\times}$. The coherence axiom for the tensor structure becomes the 2-coboundary condition

$$
\begin{aligned}
\phi(y, z) \phi(x+y, z)^{-1} \phi(x, y+z)^{-1} \phi(x, y)^{-1} & \\
& =\omega(x, y, z) \omega^{\prime}(f(x), f(y), f(z))^{-1},
\end{aligned}
$$

for all $x, y, z \in A$. Here $\omega, \omega^{\prime}$ are the associativity constraints in $\mathscr{C}, \mathscr{C}^{\prime}$ respectively. The tensor functor $F$ is braided if

$$
c(x, y) c^{\prime}(f(x), f(y))^{-1}=\phi(x, y) \phi(y, x)^{-1} .
$$


Tensor autoequivalences isomorphic to the identity functor (identity $f$ ) define an equivalence relation on the group of pairs $(\omega, c)$, where $(\omega, c)$ and $\left(\omega^{\prime}, c^{\prime}\right)$ are related as in (9) and (10) with trivial $f$. The quotient group is known as the third abelian cohomology $H_{a b}^{3}\left(A, k^{\times}\right)$[Eilenberg and Mac Lane 1954]. Elements of the latter group parametrize equivalence classes of pointed braided fusion categories.

The function

$$
q(x):=c(x, x), \quad x \in A
$$

is a quadratic form on $A$, that is, $q(-x)=q(x)$ and the symmetric function

$$
\sigma(x, y)=\frac{q(x+y)}{q(a) q(b)}, \quad x, y \in A
$$

is bimultiplicative. We have the identity

$$
\sigma(x, y)=c(x, y) c(y, x), \quad x, y \in A .
$$

Mac Lane proved that the map $(\omega, c) \mapsto q$ defines an isomorphism between $H_{a b}^{3}\left(A, k^{*}\right)$ and the group of quadratic forms $A \rightarrow k^{\times}$.

By associating to $\mathscr{C}$ the pair $(A, q)$ one gets a functor from the 1-categorical contraction of the 2-category of pointed braided fusion categories to the category of premetric groups. Each objects of the latter category is a finite abelian group equipped with a quadratic form, and the morphisms are group homomorphisms preserving the quadratic forms (that is, orthogonal homomorphisms).

It was proved by Joyal and Street [1993] that the above functor is an equivalence (see also [Drinfeld et al. 2010, Appendix D]). The braided fusion category associated to $(A, q)$ will be denoted $\mathscr{C}(A, q)$.

It follows from the above that

$$
\operatorname{Aut}^{\mathrm{br}}(\mathscr{C}(A, q))=O(A, q),
$$

where $O(A, q)$ denotes the group of orthogonal automorphisms of $(A, q)$, that is, automorphisms $\alpha: A \rightarrow A$ such that $q \circ \alpha=q$.

\section{D. Centralizers in braided tensor categories.}

Definition 2.3 (M. Müger [2003]). Two objects $X$ and $Y$ of a braided tensor category $\mathscr{C}$ are said to centralize each other if

$$
c_{Y, X} c_{X, Y}=\operatorname{id}_{X \otimes Y} .
$$

The centralizer $\mathscr{D}^{\prime}$ of a tensor subcategory $\mathscr{D} \subset \mathscr{C}$ is defined to be the full subcategory of objects of $\mathscr{C}$ that centralize each object of $\mathscr{D}$. It is easy to see that $\mathscr{D}^{\prime}$ is a tensor subcategory. 
We will denote the self-centralizer $\mathscr{C}^{\prime}$ of $\mathscr{C}$ by $\mathscr{L}_{\text {sym }}(\mathscr{C})$ and call it the symmetric center of $\mathscr{C}$. We say that $\mathscr{C}$ is nondegenerate if and only if $\mathscr{L}_{\text {sym }}(\mathscr{C})$ is trivial, that is, consists of extensions of the unit object 1.

Remark 2.4. It was shown in [Drinfeld et al. 2010, Proposition 3.7] that a braided fusion category $\mathscr{C}$ is nondegenerate if and only if it is factorizable.

Let $\mathscr{C}$ be a braided tensor category. Let us identify $\mathscr{C}$ and $\mathscr{C}^{\text {rev }}$ with their images in $\mathscr{L}(\mathscr{C})$ under the embeddings (4). Then $\mathscr{C}$ and $\mathscr{C}^{\text {rev }}$ are centralizers of each other.

Example 2.5. Let us describe the centralizers in the pointed braided fusion category $\mathscr{C}(A, q)$, see Example 2.2. Two simple objects $x, y \in A$ of this category centralize each other if and only if $\sigma(x, y)=1$, where $\sigma$ is the bimultiplicative symmetric function (11) corresponding to $q$. That is, in this case the centralizing property coincides with orthogonality.

Every fusion subcategory of $\mathscr{C}(A, q)$ corresponds to a subgroup $B \subset A$ and is equivalent to $\mathscr{C}\left(B,\left.q\right|_{B}\right)$. We have $\mathscr{C}\left(B,\left.q\right|_{B}\right)^{\prime}=\mathscr{C}\left(B^{\perp}, q_{\mid} B^{\perp}\right)$, where $B^{\perp}$ is the subgroup of $A$ orthogonal to $B$. In particular,

$$
\mathscr{L}_{\text {sym }}(\mathscr{C}(A, q))=\mathscr{C}\left(A^{\perp},\left.q\right|_{A^{\perp}}\right),
$$

where $A^{\perp}=\{a \in A \mid \sigma(a, b)=1$ for all $b \in A\}$ is the kernel of $\sigma$. The category $\mathscr{C}(A, q)$ is nondegenerate if and only if $\sigma$ is nondegenerate.

2E. Module categories over tensor categories. Let $\mathscr{A}$ be a finite tensor category. A left $\mathscr{A}$-module category (see [Quillen 1973; Janelidze and Kelly 2001; Ostrik 2003b]) is a finite category $\mathcal{M}$ together with a bifunctor

$$
\mathscr{A} \times M \rightarrow \mathcal{M}, \quad(X, M) \mapsto X * M
$$

equipped with a functorial isomorphism

$$
a_{X, Y, M}: X *(Y * M) \stackrel{\simeq}{\rightarrow}(X \otimes Y) * M, \quad X, Y \in \mathscr{A}, M \in \mathcal{M},
$$

called the associativity constraint, plus a unit constraint, the whole satisfying natural compatibility axioms.

Equivalently, $\mathcal{M}$ is a left module category over $\mathscr{A}$ if there is given a tensor functor $\mathscr{A} \rightarrow \operatorname{End}(\mathcal{M})$ to the tensor category $\operatorname{End}(\mathcal{M})$ of endofunctors of $\mathcal{M}$ (with tensor structure given by composition of functors).

A right $\mathscr{A}$-module category is defined in a similar way. It corresponds to a tensor functor $\mathscr{A}^{\mathrm{op}} \rightarrow \operatorname{End}(\mathcal{M})$. For a right $\mathscr{A}$-module category $\mathcal{M}$ the category obtained from $\mathcal{M}$ reversing the directions of morphisms is a left $\mathscr{A}$-module category via

$$
X \odot M=M * X^{*}, \quad M \in M, X \in \mathscr{A} .
$$

We will denote this category $M^{\mathrm{op}}$ and call it the opposite module category. 
Functors between $\mathscr{A}$-module categories and natural transformations between them are defined in an obvious way, see [Ostrik 2003b].

Let $\mathscr{A}$ be a tensor category. Following [Etingof and Ostrik 2004] we say that an $\mathscr{A}$-module category $\mathcal{M}$ is exact if for any projective object $P$ of $\mathscr{A}$ and every object $M$ of $\mathcal{M}$ the object $P \otimes M$ is projective. An $\mathscr{A}$-module category $\mathcal{M}$ is exact if and only if for every $\mathscr{C}$-module category $\mathcal{N}$ any $\mathscr{C}$-module functor $\mathcal{M} \rightarrow \mathcal{N}$ is exact.

Example 2.6. If $\mathscr{A}$ is a fusion category then an $\mathscr{A}$-module category is exact if and only if it is semisimple.

Note 2.7. All module categories in this paper are assumed to be exact.

Given an indecomposable left $\mathscr{A}$-module category $\mathcal{M}$ the $d u a l$ category of $\mathscr{A}$ with respect to $\mathcal{M}$ is the category $\mathscr{A}_{\mathcal{M}}^{*}=\operatorname{Fun}_{\mathscr{A}}(\mathcal{M}, \mathcal{M})$ of $\mathscr{A}$-module endofunctors of $\mathcal{M}$. It was shown in [Etingof and Ostrik 2004, Section 3.3] that $\mathscr{A}_{\mathcal{M}}^{*}$ is a finite tensor category. Furthermore, $\mathcal{M}$ is an exact indecomposable left $\mathscr{A}_{\mathcal{M}}^{*}$-module category and there is a canonical tensor equivalence $\mathscr{A} \cong\left(\mathscr{A}_{M}^{*}\right)_{\mathcal{M}}^{*}$.

Remark 2.8. It was proved in [Etingof and Ostrik 2004, Theorem 3.31] that the assignment

$$
\mathcal{N} \mapsto \operatorname{Fun}_{\mathscr{A}}(\mathcal{M}, \mathcal{N})
$$

is an equivalence between the 2 -category of exact left $\mathscr{A}$-module categories and that of exact right $\mathscr{A}_{\mathcal{M}}^{*}$-module categories.

2F. Bimodule categories. Let $\mathscr{A}, \mathscr{B}$ be tensor categories.

By definition, an $(\mathscr{A}-\mathscr{B})$-bimodule category $\mathcal{M}$ is an $\left(\mathscr{A} \bigotimes \mathscr{B}^{\mathrm{op}}\right)$-module category.

Equivalently, a category $\mathcal{M}$ is an $(\mathscr{A}-\mathscr{B})$-bimodule category if it has left $\mathscr{A}$ module and right $\mathscr{B}$-module category structures compatible by a collection of isomorphisms $a_{X, M, Y}: X *(M * Y) \rightarrow(X * M) * Y$ called middle associativity constraints natural in $X \in \mathscr{A}, Y \in \mathscr{B}, M \in \mathcal{M}$, and such that the diagrams

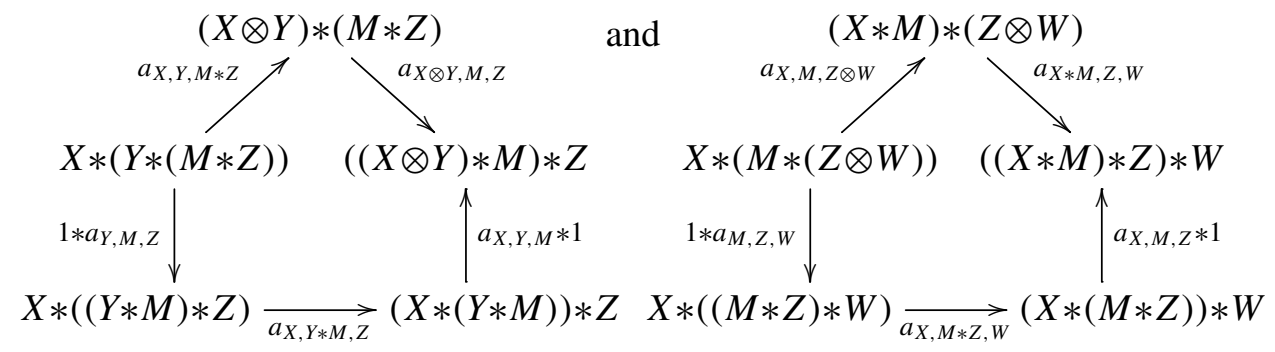

commute for all $X, Y \in \mathscr{A}, Z, W \in \mathscr{B}$, and $M \in \mathcal{M}$.

Example 2.9. A left $\mathscr{A}$-module category $\mathcal{M}$ has a structure of an $\left(\mathscr{A}-\left(\mathscr{A}_{\mathcal{M}}^{*}\right)^{\text {op }}\right)$ bimodule category. 
2G. Tensor product of module categories and the Brauer-Picard group of a tensor category. Let $\mathscr{A}$ be a finite tensor category, let $\mathcal{M}$ be a right $\mathscr{A}$-module category, and let $\mathcal{N}$ be a left $\mathscr{A}$-module category. The $\mathscr{A}$-module tensor product of $\mathcal{M}$ and $\mathcal{N}$ was defined in [Etingof et al. 2010, Section 3.1]. Let us recall this definition. A bifunctor $F: \mathcal{M} \times \mathcal{N} \rightarrow \mathscr{K}$, where $\mathscr{K}$ is an abelian category, is called $\mathscr{A}$-balanced if there exists a family of isomorphisms $F(M \otimes X, N) \stackrel{\widetilde{ }}{\rightarrow} F(M, X \otimes N)$ natural in $M \in \mathcal{M}, N \in \mathcal{N}$, and $X \in \mathscr{A}$ satisfying coherence axioms. Let $\operatorname{Fun}_{b a l, r e}(M \times \mathcal{N}, \mathscr{K})$ denote the category of $\mathscr{A}$-balanced functors from $M \times \mathcal{N}$ to $\mathscr{K}$ right exact in each variable.

The $\mathscr{A}$-module tensor product of $\mathcal{M}$ and $\mathcal{N}$ is an abelian category $\mathcal{M} \bigotimes_{\mathscr{A}} \mathcal{N}$ together with the $\mathscr{b}$-balanced bifunctor

$$
B_{\mathcal{M}, \mathcal{N}}: M \times \mathcal{N} \rightarrow M \otimes_{\mathscr{A}} \mathcal{N}
$$

which is right exact in each variable and for every abelian category $\mathscr{K}$ induces an equivalence

$$
\operatorname{Fun}_{b a l, r e}(\mathcal{M} \times \mathcal{N}, \mathscr{A}) \simeq \operatorname{Fun}_{r e}\left(\mathcal{M} \bigotimes_{\mathscr{A}} \mathcal{N}, \mathscr{K}\right)
$$

Here and below, the subscript $r e$ indicates that functors under consideration are right exact. The existence of the $\mathscr{A}$-module tensor product was established in [Etingof et al. 2010, Section 3.2]. Namely, it was shown that

$$
M \otimes_{\mathscr{A}} \mathcal{N} \simeq \operatorname{Fun}_{\mathscr{A}, r e}\left(M^{\mathrm{op}}, \mathcal{N}\right) .
$$

Note that although the categories considered in [Etingof et al. 2010] were assumed to be semisimple the proof of this particular result does not use semisimplicity. Indeed, first observe that $\mathcal{M} \otimes \mathcal{N}$ is equivalent to $\operatorname{Fun}_{r e}\left(M^{\text {op }}, \mathcal{N}\right)$, since for $\mathcal{M}=\operatorname{Rep}(A)$ and $\mathcal{N}=\operatorname{Rep}(B)$, where $A$ and $B$ are algebras, both categories are identified with $\operatorname{Rep}(A \otimes B)$. Next, by [Etingof et al. 2010, Proposition 3.5] every balanced bifunctor $\mathcal{M} \times \mathcal{N} \rightarrow \mathscr{K}$ that is right exact in every variable canonically factors through the functor

$$
\mathcal{M} \otimes \mathcal{N} \simeq \operatorname{Fun}_{r e}\left(\mathcal{M}^{\mathrm{op}}, \mathcal{N}\right) \stackrel{B_{\mathcal{M}, \mathcal{N}}}{\longrightarrow} \operatorname{Fun}_{\mathscr{A}, r e}\left(\mathcal{M}^{\mathrm{op}}, \mathcal{N}\right)
$$

where $B_{\mathcal{M}, \mathcal{N}}$ is the left adjoint to the forgetful functor

$$
\operatorname{Fun}_{\mathscr{A}, r e}\left(\mathcal{M}^{\mathrm{op}}, \mathcal{N}\right) \rightarrow \operatorname{Fun}_{r e}\left(\mathcal{M}^{\mathrm{op}}, \mathcal{N}\right)
$$

Furthermore, if $\mathcal{M}$ and $\mathcal{N}$ are $\mathscr{A}$-bimodule categories then so is $M \nabla_{\mathscr{A}} \mathcal{N}$ (the $\mathscr{A}$-bimodule structure on $\mathcal{M} \bigotimes_{\mathscr{A}} \mathcal{N}$ is induced by the $\mathscr{A}$-bimodule structure on $M \otimes \mathcal{N}$ ).

Proposition 2.10. Let $\mathcal{M}$ and $\mathcal{N}$ be exact $\mathscr{A}$-bimodule categories. Then $\mathcal{M} \nabla_{\mathscr{A}} \mathcal{N}$ is an exact $\mathscr{A}$-bimodule category. 
Proof. It is enough to check that for all objects $F$ in $M \nabla_{\mathscr{A}} \mathcal{N}$ and projective objects $P_{1}, P_{2}$ in $\mathscr{C}$ the object $P_{1} \otimes F \otimes P_{2}$ is projective. That is, we need to show that the compositions of an $\mathscr{A}$-module functor $F: M^{\text {op }} \rightarrow \mathcal{N}$ with the functors

$$
\begin{gathered}
\mu^{\mathrm{op}} \rightarrow \mathcal{M}^{\mathrm{op}}: M \mapsto M \otimes P_{1}, \\
\mathcal{N} \rightarrow \mathcal{N}: N \mapsto N \otimes P_{2}
\end{gathered}
$$

are projective objects in $\operatorname{Fun}_{\mathscr{A}}\left(\mathcal{M}^{\mathrm{op}}, \mathcal{N}\right)$. This is clear since the latter category is exact over $\mathscr{A}_{\mathcal{M}}^{*}$ and $\mathscr{A}_{\mathcal{N}}^{*}$ and the right multiplications by $P_{1}, P_{2}$ are $\mathscr{A}$-module endofunctors.

We say that an exact $\mathscr{A}$-bimodule category $\mathcal{M}$ is invertible if there exists an exact $\mathscr{A}$-bimodule category $\mathcal{N}$ such that

$$
M \nabla_{\mathscr{A}} \mathcal{N} \simeq \mathcal{N} \nabla_{\mathscr{A}} \mathcal{M} \simeq \mathscr{A}
$$

where $\mathscr{A}$ is viewed as an $\mathscr{A}$-bimodule category via the regular left and right actions of $\mathscr{A}$.

Remark 2.11. It was proved in [Etingof et al. 2010, Propositon 4.2] that an $\mathscr{A}$ bimodule category $\mathcal{M}$ is invertible if and only if the tensor functor

$$
L: \mathscr{A} \rightarrow\left(\mathscr{A}_{M}^{*}\right)^{\mathrm{op}}: X \mapsto ? \otimes X
$$

is an equivalence.

The group of equivalence classes of invertible $\mathscr{A}$-bimodule categories is called the Brauer-Picard group of $\mathscr{A}$ and is denoted by $\operatorname{BrPic}(\mathscr{A})$.

2H. Module categories over braided tensor categories. Let now $\mathscr{C}$ be a braided tensor category with the braiding

$$
c_{X, Y}: X \otimes Y \stackrel{\simeq}{\rightarrow} Y \otimes X, \quad X, Y \in \mathscr{C} .
$$

The braiding of $\mathscr{C}$ gives a tensor structure on the multiplication functor $\mathscr{C} \nabla \mathscr{C} \rightarrow \mathscr{C}$ [Joyal and Street 1993]. Hence, there is a canonical tensor functor

$$
\otimes: \mathscr{C} \nabla \mathscr{C}^{\mathrm{op}} \simeq \mathscr{C} \nabla \mathscr{C} \rightarrow \mathscr{C} .
$$

This allows us to turn any left $\mathscr{b}$-module category $\mathcal{M}$ into a $\mathscr{b}$-bimodule category as follows. The right action is $M * X:=X * M$ for all $X \in \mathscr{C}$ and $M \in M$. Let $a_{X, Y, M}: X \otimes(Y \otimes M) \stackrel{\simeq}{\rightarrow}(X \otimes Y) \otimes M$ denote the left $\mathscr{C}$-module associativity constraint of $\mathcal{M}$. The right $\mathscr{b}$-module associativity constraint of $\mathcal{M}$ is given by

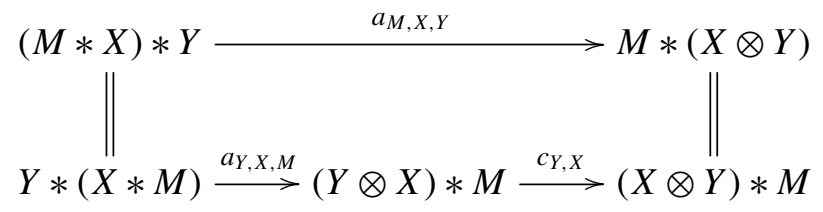


and the middle associativity constraint is given by

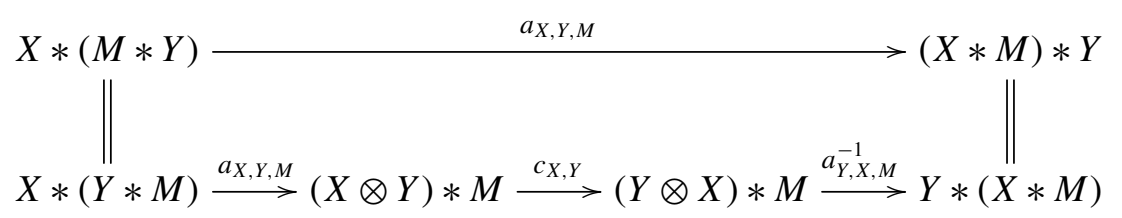

for all $X, Y \in \mathscr{C}$ and $M \in \mathcal{M}$.

Let $\operatorname{Mod}(\mathscr{C})$ and $\operatorname{Bimod}(\mathscr{C})$ denote the 2-categories of exact module and bimodule categories over $\mathscr{C}$, respectively. The above tensor functor (15) yields a 2-functor

$$
\mathscr{B}: \operatorname{Mod}(\mathscr{C}) \rightarrow \operatorname{Bimod}(\mathscr{C})
$$

Clearly, the 2-functor $\mathscr{B}$ is an embedding of 2-categories.

Definition 2.12. We will call a $\mathscr{C}$-bimodule category one-sided if it is equivalent to $\mathscr{B}(\mathcal{M})$ for some left $\mathscr{C}$-module category $\mathcal{M}$.

Remark 2.13. One can give an explicit characterization of one-sided categories. Namely, a $\mathscr{C}$-bimodule category $\mathcal{M}$ is one-sided if it is equipped with a collection of isomorphisms

$$
d_{M, X}: M * X \rightarrow X * M
$$

natural in $X \in \mathscr{C}$ and $M \in \mathcal{M}$, such that the diagrams

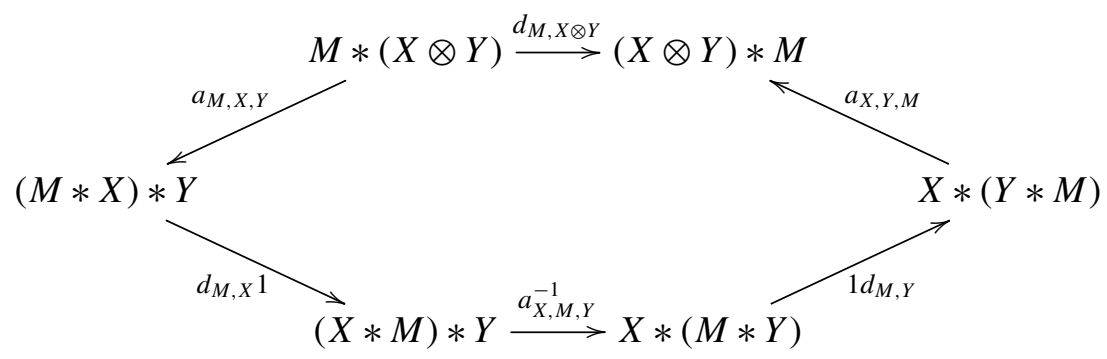

and

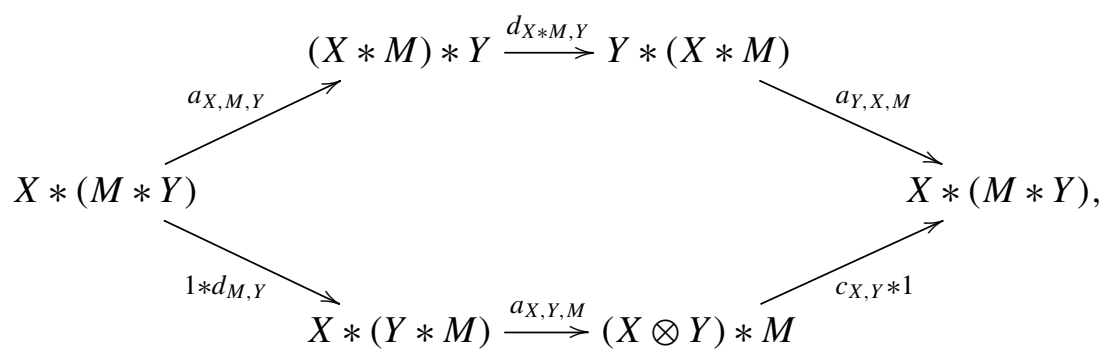

commute, where $a$ denotes the associativity constraint of $\mathcal{M}$. 
Given left $\mathscr{b}$-module categories $\mathcal{M}$ and $\mathcal{N}$, there is an obvious $\mathscr{b}$-bimodule equivalence

$$
\mathscr{B}\left(\mathscr{B}(\mathcal{M}) \nabla_{\mathscr{C}} \mathcal{N}\right) \simeq \mathscr{B}(\mathcal{M}) \otimes_{\mathscr{C}} \mathscr{B}(\mathcal{N})
$$

Hence, when $\mathscr{b}$ is braided, the group $\operatorname{BrPic}(\mathscr{C})$ contains a subgroup $\operatorname{Pic}(\mathscr{C})$ consisting of equivalence classes of one-sided invertible $\mathscr{C}$-bimodule categories. Following [Etingof et al. 2010], we call this group the Picard group of $\mathscr{C}$.

In what follows we will omit the 2 -functor $\mathscr{B}$ from notation and identify invertible $\mathscr{C}$-module categories with their images in $\operatorname{Bimod}(\mathscr{C})$.

2I. The $\alpha$-induction. Let $\mathscr{C}$ be a braided tensor category and let $\mathcal{M}$ be a $\mathscr{C}$-module category. There is a pair of tensor functors

$$
\alpha_{\mathcal{M}}^{ \pm}: \mathscr{C} \rightarrow \mathscr{C}_{\mathcal{M}}^{*}
$$

defined as follows (see [Böckenhauer et al. 2001; Ostrik 2003b]). For each $X \in \mathscr{C}$ the endofunctors $\alpha_{\mathcal{M}}^{ \pm}(X): \mathcal{M} \rightarrow \mathcal{M}$ coincide with left multiplication by $X$, that is,

$$
\alpha_{M}^{ \pm}(X)=X \otimes-
$$

Their $\mathscr{b}$-module functor structures are given by

$$
\begin{aligned}
& \alpha_{\mathcal{M}}^{+}(X)(Y \otimes M)=X \otimes Y \otimes M \stackrel{c_{X, Y}}{\longrightarrow} Y \otimes X \otimes M=Y \otimes \alpha_{\mathcal{M}}^{+}(X)(M), \\
& \alpha_{\mathcal{M}}^{-}(X)(M \otimes Y)=X \otimes Y \otimes M \stackrel{c_{Y, X}^{-1}}{\longrightarrow} Y \otimes X \otimes M=Y \otimes \alpha_{\mathcal{M}}^{-}(X)(M),
\end{aligned}
$$

for all $X, Y \in \mathscr{C}$ and $M \in M$. Here we suppress the associativity constraints.

When $\mathcal{M}$ is invertible the functors $\alpha_{\mathcal{M}}^{ \pm}$are equivalences and the functor $\partial_{\mathcal{M}}: \mathscr{C} \rightarrow \mathscr{C}$ defined by

$$
\left(\alpha_{\mathcal{M}}^{-}\right) \circ \partial_{\mathcal{M}}=\alpha_{\mathcal{M}}^{+}
$$

is a braided autoequivalence of $\mathscr{C}$. The assignment $\mathcal{M} \mapsto \partial_{\mu}$ gives rise to a group homomorphism

$$
\partial: \operatorname{Pic}(\mathscr{C}) \rightarrow \operatorname{Aut}^{\mathrm{br}}(\mathscr{C}), \quad \mu \mapsto \partial_{\mu}
$$

To be precise, the condition (23) defines a tensor autoequivalence of $\mathscr{C}$. The reason why it is braided is explained in Remark 4.5 (see also [Etingof et al. 2010] for details in the fusion case).

\section{The Picard crossed module of a braided tensor category}

3A. Algebras and their modules. We refer the reader to [Ostrik 2003b] for basic definitions and facts about algebras in tensor categories and modules over them. 
Let $A$ be an algebra in a tensor category $\mathscr{A}$ with the multiplication $\mu: A \otimes A \rightarrow A$ and let $M$ be a right $A$-module in $\mathscr{A}$ with the structural map $v: M \otimes A \rightarrow M$. For any $X \in \mathscr{A}$ there is an $A$-module structure on $X \otimes M$ defined by

$$
\operatorname{id}_{X} \otimes v: X \otimes M \otimes A \rightarrow X \otimes M .
$$

Thus the category $\mathscr{A}_{A}$ of right $A$-modules in $\mathscr{A}$ is a left $\mathscr{A}$-module category via

$$
\mathscr{A} \times \mathscr{A}_{A} \rightarrow \mathscr{A}_{A}, \quad(X, M) \mapsto X \otimes M .
$$

Similarly, the category ${ }_{A} \mathscr{A}$ of left $A$-modules in $\mathscr{A}$ is a right $\mathscr{A}$-module category.

We say that an algebra $A$ is exact if the $\mathscr{A}$-module categories $\mathscr{A}_{A}$ and ${ }_{A} \mathscr{A}$ are exact.

Remark 3.1. Let $A$ be an algebra in $\mathscr{A}$. Then the left $\mathscr{A}$-module category $\left({ }_{A} \mathscr{A}\right)^{\mathrm{op}}$ is equivalent to $\mathscr{A}_{A}$.

It was shown in [Etingof and Ostrik 2004] that every left (respectively, right) $\mathscr{A}$-module category is equivalent to $\mathscr{A}_{A}$ (respectively, to ${ }_{A} \mathscr{A}$ ) for some algebra $A$ in A.

Let $A$ be an algebra in a tensor category $\mathscr{A}$ and $\mathcal{M}$ be a left $\mathscr{A}$-module category. Define ${ }_{A} \mathcal{M}$ (the category of $A$-modules in $\mathcal{M}$ ) as the category of pairs $(M, m)$, where $M$ is an object of $\mathcal{M}$ and $m: A * M \rightarrow M$ is a morphism in $M$ such that the diagram

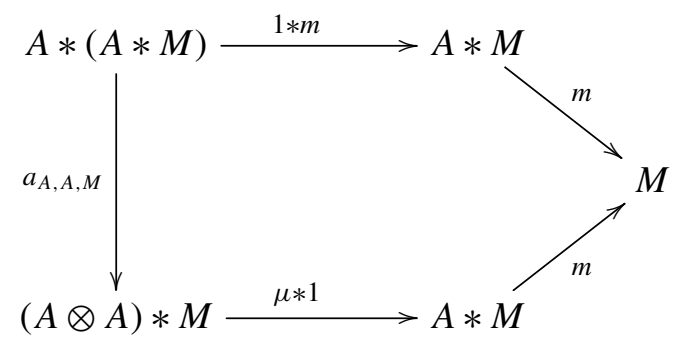

commutes.

A morphism between $(M, m)$ and $\left(M^{\prime}, m^{\prime}\right)$ is a morphism $f: M \rightarrow M^{\prime}$ such that $f \circ m=m^{\prime} \circ\left(\mathrm{id}_{A} * f\right)$.

Lemma 3.2. Let $\mathscr{A}$ be a finite tensor category and let $M$ be an exact right $\mathscr{A}$-module category. The functor

$$
T: \operatorname{Fun}_{\mathscr{A}}\left(\mathscr{A}_{A}, \mathcal{M}\right) \rightarrow{ }_{A} \mathcal{M}: F \mapsto F(A)
$$

is an equivalence of categories.

Proof. For any $\mathscr{A}$-module functor $F: \mathscr{A}_{A} \rightarrow \mathcal{M}$ the object $F(A) \in \mathcal{M}$ has a structure of an $A$-module,

$$
A * F(A) \stackrel{\simeq}{\rightarrow} F(A \otimes A) \stackrel{F(\mu)}{\longrightarrow} F(A)
$$


where the first arrow is given by the $\mathscr{A}$-module structure of $F$ and the second arrow is the image of the multiplication of $A$. It is easy to see that $\mathscr{A}$-module transformations between $\mathscr{A}$-module functors $F, G$ correspond to morphisms of $A$-modules $F(A), G(A)$ in $M$. Thus, $T$ is a well-defined functor.

Define a functor $S:{ }_{A} \mathcal{M} \rightarrow \operatorname{Fun}_{\mathscr{A}}\left(\mathscr{A}_{A}, \mathcal{M}\right)$ by $M \mapsto S_{M}$, where $S_{M}(X)=X \otimes_{A} M$. It is clear that $S_{M}$ is an $\mathscr{A}$-module functor and that $T \circ S$ is isomorphic to the identity endofunctor of ${ }_{A} \mathcal{M}$.

Also, $S \circ T$ is isomorphic to the identity functor since for every $\mathscr{A}$-module functor $F: \mathscr{A}_{A} \rightarrow \mathcal{M}$ and a right $A$-module $X$ in $\mathscr{A}$ there is a natural isomorphism $X \otimes_{A} F(A) \cong F(X)$. Thus, $T$ is an equivalence.

A particular case of Lemma 3.2 that will be useful for us later is the category of $A$-modules in $\mathcal{M}=\mathscr{A}_{B}$, where $B$ is an exact algebra in $\mathscr{A}$. The category ${ }_{A} \mathscr{A}_{B}$ is the category of $(A-B)$-bimodules in $\mathscr{C}$.

Corollary 3.3. The functor

$$
\operatorname{Fun}_{\mathscr{A}}\left(\mathscr{A}_{A}, \mathscr{A}_{B}\right) \rightarrow{ }_{A} \mathscr{A}_{B}, \quad F \mapsto F(A)
$$

is an equivalence of categories.

3B. Tensor product of algebras in a braided category. Let now $\mathscr{C}$ be a braided tensor category and let $A$ be an algebra in $\mathscr{C}$. Given a left $\mathscr{C}$-module category $\mathcal{M}$, the braiding in $\mathscr{C}$ allows us to turn ${ }_{A} \mathcal{M}$ into a left $\mathscr{C}$-module category. In this situation the functor $\operatorname{Fun}_{\mathscr{C}}\left(\mathscr{C}_{A}, \mathcal{M}\right) \stackrel{\sim}{\rightarrow}{ }_{A} \mathcal{M}$ from Lemma 3.2 is an equivalence of $\mathscr{b}$-module categories.

It is well-known that for braided $\mathscr{C}$ the tensor product $A \otimes B$ of two algebras $A, B \in \mathscr{C}$ has an algebra structure, with the multiplication map $\mu_{A \otimes B}$ defined as

$$
A \otimes B \otimes A \otimes B \stackrel{\mathrm{id}_{A} \otimes c_{B, A} \otimes \mathrm{id}_{B}}{\longrightarrow} A \otimes A \otimes B \otimes B \stackrel{\mu_{A} \otimes \mu_{B}}{\longrightarrow} A \otimes B,
$$

where $\mu_{A}$ and $\mu_{B}$ are multiplications of algebras $A$ and $B$, respectively (here we suppress the associativity constraints in $\mathscr{C}$ ).

Let $A^{\mathrm{op}}=A$ denote the algebra with the multiplication opposite to that of $A$ :

$$
A \otimes A \stackrel{c_{A, A}}{\longrightarrow} A \otimes A \stackrel{\mu_{A}}{\longrightarrow} A .
$$

Proposition 3.4. Let $\mathscr{C}$ be a braided tensor category and let $A$ and $B$ be exact algebras in $\mathcal{C}$. Then

$$
\mathscr{C}_{A} \otimes_{\mathscr{C}} \mathscr{b}_{B} \simeq \mathscr{C}_{A \otimes B}
$$

as $\mathscr{C}$-module categories.

Proof. Note that a left $\mathscr{b}$-module category $\mathscr{C}_{A}$ considered as a right $\mathscr{b}$-module

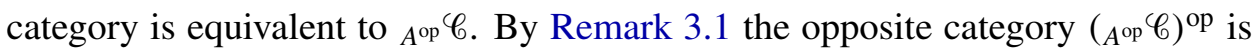
equivalent to $\mathscr{C}_{A^{\text {op }}}$ as a left $\mathscr{b}$-module category. 
Hence, using (13) and Corollary 3.3 we obtain $\mathscr{C}_{A} \nabla_{\mathscr{C}_{C}} \mathscr{C}_{B} \simeq \operatorname{Fun}_{\mathscr{C}}\left(\left(A^{\text {op }} \mathscr{C}^{\text {op }}, \mathscr{C}_{B}\right) \simeq\right.$ Fun $_{\mathscr{C}}\left(\mathscr{C}_{A^{\text {op }}}, \mathscr{C}_{B}\right) \simeq{ }_{A^{\text {op }}} \mathscr{C}_{B} \simeq \mathscr{C}_{A \otimes B}$, since an $(A \otimes B)$-module in $\mathscr{C}_{\text {is }}$ the same thing as an $\left(A^{\text {op }}-B\right)$-bimodule.

3C. Azumaya algebras. Here we recall the characterization of algebras in $\mathscr{b}$ whose categories of modules are invertible.

Let $A$ be an exact algebra in a braided tensor category $\mathscr{C}$.

Note that multiplication on $A$, via

$$
A \otimes A^{\mathrm{op}} \otimes A \stackrel{\mathrm{id}_{A} \otimes c_{A, A}}{\longrightarrow} A \otimes A \otimes A \stackrel{\mu_{A} \otimes \mathrm{id}_{A}}{\longrightarrow} A \otimes A \stackrel{\mu_{A}}{\longrightarrow} A,
$$

induces a homomorphism of algebras

$$
A \otimes A^{\mathrm{op}} \rightarrow A \otimes A^{*}
$$

where $A^{*}$ is the dual object to $A$ and the multiplication in $A \otimes A^{*}$ is defined using the evaluation morphism.

Definition 3.5. An exact algebra $A$ in a braided tensor category $\mathscr{C}$ is Azumaya if the map (27) is an isomorphism.

It was established in [Van Oystaeyen and Zhang 1998, Theorem 3.1] that $A$ is an Azumaya algebra if and only if the tensor functors

$$
\alpha_{\mathscr{C}_{A}}^{ \pm}: \mathscr{C} \rightarrow{ }_{A} \mathscr{C}_{A}
$$

defined in (22) are equivalences. Thus, the Picard group of $\mathscr{C}$ is isomorphic to the group of Morita equivalence classes of Azumaya algebras (the latter group was considered in [Van Oystaeyen and Zhang 1998]).

Let $A$ be an Azumaya algebra in $\mathscr{C}$. Let $\partial_{A}=\partial_{\mathscr{C}_{A}}$ denote the braided autoequivalence introduced in (24). By definition of $\partial_{A}$, there exists a natural isomorphism of right $A$-modules

$$
\phi_{X}: A \otimes X \stackrel{\simeq}{\rightarrow} \partial_{A}(X) \otimes A, \quad X \in \mathscr{C} .
$$

This means that the following diagram commutes:

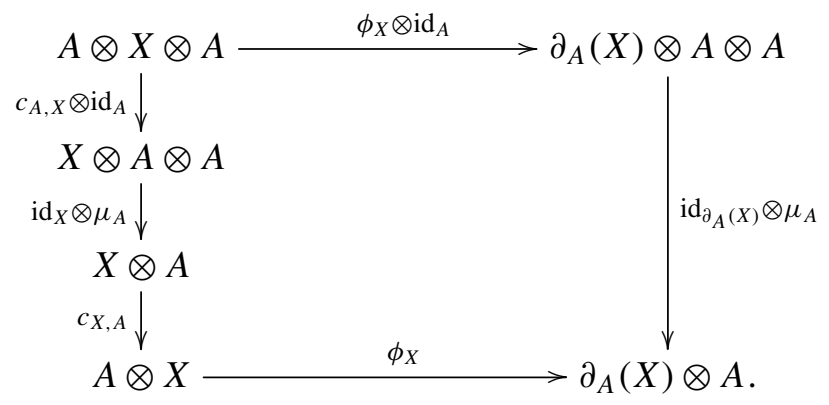


The tensor structure

$$
v_{X, Y}: \partial_{A}(X \otimes Y) \stackrel{\simeq}{\rightarrow} \partial_{A}(X) \otimes \partial_{A}(Y), \quad X, Y \in \mathscr{C}
$$

of $\partial_{A}$ satisfies the following commutative diagram:

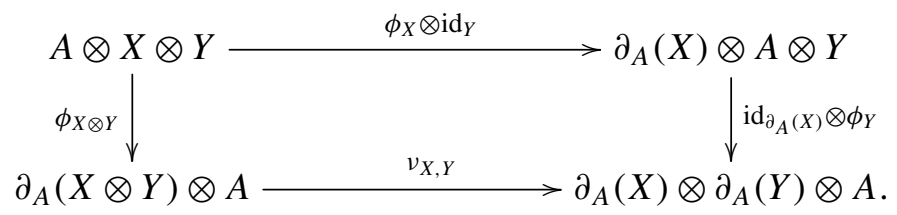

Lemma 3.6. The diagram

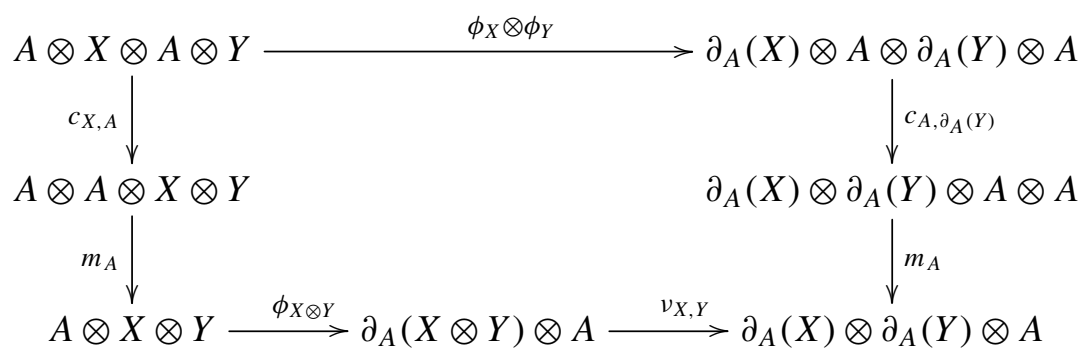

is commutative (here, as usual, we suppress the associativity constraints and identity morphisms).

Proof. Note that compositions of the left and the right vertical arrows in diagram (30) coincide, respectively, with the canonical epimorphisms

$$
A \otimes X \otimes A \otimes Y \rightarrow(A \otimes X) \otimes_{A}(A \otimes Y) \cong A \otimes X \otimes Y
$$

and

$\partial_{\mathscr{A}}(X) \otimes A \otimes \partial_{\mathscr{A}}(Y) \otimes A \rightarrow\left(\partial_{A}(X) \otimes A\right) \otimes_{A}\left(\partial_{A}(Y) \otimes A\right) \cong \partial_{\mathscr{A}}(X) \otimes \partial_{\mathscr{A}}(Y) \otimes A$. Hence, the diagram

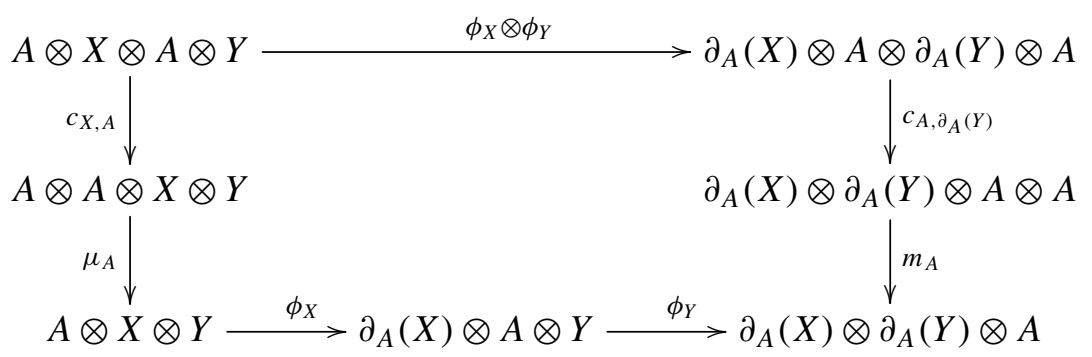

is commutative by functoriality of $\otimes_{A}$. But the bottom row composition in diagram (31) coincides with that of diagram (30) by the identity (29). 
Let $B$ be an algebra in $\mathscr{C}$ and suppose that $A$ is an Azumaya algebra in $\mathscr{C}$. Then $\partial_{A}(B)$ is also an algebra in $\mathscr{C}$. We will denote by

$$
\mu_{B}: B \otimes B \rightarrow B \quad \text { and } \quad \mu_{\partial_{A}(B)}: \partial_{A}(B) \otimes \partial_{A}(B) \rightarrow \partial_{A}(B)
$$

the multiplications of $B$ and $\partial_{A}(B)$ respectively.

Proposition 3.7. The morphism $\phi_{B}: A \otimes B \rightarrow \partial_{A}(B) \otimes A$ is an isomorphism of algebras.

Proof. Consider the diagram

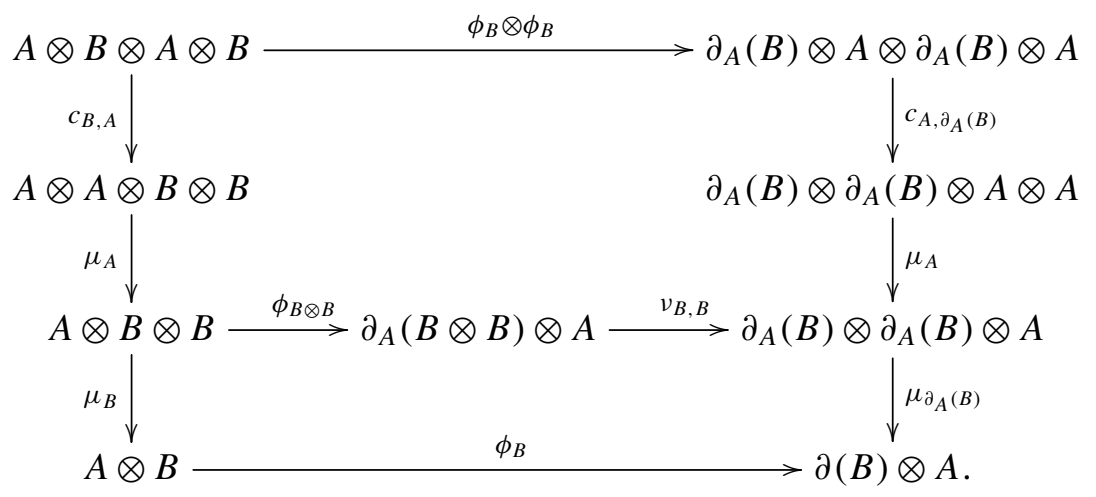

The upper subdiagram is commutative by Lemma 3.6 and the lower subdiagram is the definition of multiplication $\mu_{\partial_{A}(B)}$. Hence, diagram (32) is commutative. This is precisely the property of $\phi_{B}$ being an algebra homomorphism.

\section{D. Definition of the Picard crossed module.}

Definition 3.8. A crossed module $(G, C)$ is a pair of groups $G$ and $C$ together with an action of $G$ on $C$, denoted by $(g, c) \mapsto{ }^{g} C$, and a homomorphism $\partial: C \rightarrow G$ satisfying

$$
\partial\left({ }^{g} c\right)=g \partial(c) g^{-1}
$$

and

$$
{ }^{\partial(c)} c^{\prime}=c c^{\prime} c^{-1} \quad c, c^{\prime} \in C, g \in G .
$$

Let $\left(G_{1}, C_{1}\right)$ and $\left(G_{2}, C_{2}\right)$ be crossed modules with structural maps $\partial_{1}: C_{1} \rightarrow G_{1}$ and $\partial_{1}: C_{2} \rightarrow G_{2}$. A homomorphism between these crossed modules is a pair of group homomorphisms $\gamma: G_{1} \rightarrow G_{2}$ and $\phi: \mathscr{C}_{1} \rightarrow \mathscr{C}_{2}$ such that $\partial_{2} \circ \phi=\gamma \circ \partial_{1}$ and $\phi\left({ }^{g} c\right)=\gamma(g) \phi(c)$ for all $c \in C_{1}$ and $g \in G_{1}$.

Remark 3.9. It is clear that the kernel of the homomorphism $\partial$ in Definition 3.8 is a subgroup of the center of $C$ and the image of $\partial$ is a normal subgroup of $G$.

Let $\mathscr{b}$ be a braided tensor category. Set

$$
G:=\operatorname{Aut}^{\mathrm{br}}(\mathscr{C}), \quad C:=\operatorname{Pic}(\mathscr{C}) .
$$


In (24) we defined a canonical homomorphism

$$
\partial: C \rightarrow G: \mathcal{M} \mapsto \partial_{\mu} .
$$

There is also a canonical action of $\operatorname{Aut}^{\mathrm{br}}(\mathscr{C})$ on Pic ( $\left.\mathscr{C}\right)$. Namely, for $g \in \operatorname{Aut}^{\mathrm{br}}(\mathscr{C})$ and a $\mathscr{b}$-module category $\mathcal{M}$ the category ${ }^{g} \mathcal{M}$ is defined as follows. As an abelian category, ${ }^{g} \mathcal{M}=\mathcal{M}$. The action of $\mathscr{C}$ on $\mathcal{M}$ is defined by

$$
X \odot M:=g^{-1}(X) * M \quad \text { for all } M \in M, X \in \mathscr{C} .
$$

Note that for an algebra $A \in \mathscr{C}$ the $\mathscr{C}_{\text {-module category }}{ }^{g}\left(\mathscr{C}_{A}\right)$ is equivalent to $\mathscr{C}_{g(A)}$. Here $g(A)$ is the algebra with multiplication $\mu_{g(A)}=g\left(\mu_{A}\right)$.

Theorem 3.10. The pair $(G, C)=\left(\operatorname{Aut}^{\mathrm{br}}(\mathscr{C})\right.$, Pic(C) $)$ equipped with the above structural operations is a crossed module.

Proof. To check the axiom (33), note that tensor equivalences

$$
\alpha_{g_{\mathcal{M}}}^{ \pm}: \mathscr{C} \rightarrow \mathscr{G}_{g_{\mathcal{M}}}^{*}
$$

defined in (22) satisfy $\alpha_{g_{\mathcal{M}}}^{ \pm} \cong \alpha_{\mathcal{M}}^{ \pm} \circ g^{-1}$. Hence,

$$
\partial_{g_{\mathcal{M}}} \cong\left(\alpha_{g_{\mathcal{M}}}^{-}\right)^{-1} \circ \alpha_{g_{\mathcal{M}}}^{+} \cong g \circ \partial_{\mathcal{M}} \circ g^{-1} \text { for all } \mathcal{M} \in \operatorname{Pic}(\mathscr{C}), g \in G .
$$

Let us check axiom (34). Take $\mathcal{M}, \mathcal{N} \in \operatorname{Pic}(\mathscr{C})$ and let $A$ and $B$ be algebras in $\mathscr{C}$ such that $\mathcal{M} \simeq \mathscr{C}_{A}$ and $\mathcal{N} \simeq \mathscr{C}_{B}$. By Proposition 3.4 we have

$$
\mu \otimes_{\mathscr{C}} \mathcal{N} \simeq \mathscr{C}_{A \otimes B} \quad \text { and } \quad \partial_{\mathcal{M}} \mathcal{N} \otimes_{\mathscr{C}} \mathcal{M} \simeq \mathscr{C}_{\partial_{\mathcal{M}}(B) \otimes A}
$$

Since by Proposition 3.7 the algebras $A \otimes B$ and $\partial_{\mu}(B) \otimes A$ are isomorphic, we conclude $M \nabla_{\mathscr{C}} \mathcal{N} \simeq{ }^{\partial_{M}} \mathcal{N} \nabla_{\mathscr{C}} M$, as required.

Definition 3.11. We will call the pair $\left(\operatorname{Aut}^{\mathrm{br}}(\mathscr{C})\right.$, Pic $\left.(\mathscr{C})\right)$ the Picard crossed module of $\mathscr{C}$ and denote it $\mathfrak{P}(\mathscr{C})$.

\section{Picard crossed module and braided autoequivalences of the center}

In this section we give a characterization of the Picard crossed module of a braided tensor category $\mathscr{C}$ in terms of braided autoequivalences of $\mathscr{L}(\mathscr{C})$.

4A. The Brauer-Picard group and braided autoequivalences of the center. Let $\mathcal{M}$ be an exact left $\mathscr{C}$-module category. It can be regarded as a $\left(\mathscr{C} \square \mathscr{C}_{\mathcal{M}}^{*}\right)$-module category. The following constructions are taken from [Etingof and Ostrik 2004, Section 3.4]: There are canonical equivalences

$$
a_{\mathcal{M}}: \mathscr{L}(\mathscr{C}) \stackrel{\sim}{\longrightarrow}\left(\mathscr{C} \nabla \mathscr{C}_{\mathcal{M}}^{*}\right)_{\mathcal{M}}^{*}:(Z, \gamma) \mapsto Z * ?,
$$


where the left $\mathscr{C}$-module functor structure of $a_{\mathcal{M}}(Z, \gamma)$ is given by

$$
X *(Z * M) \stackrel{a_{X, Z, M}}{\longrightarrow}(X \otimes Z) * M \stackrel{\gamma_{X}}{\longrightarrow}(Z \otimes X) * M \stackrel{a_{Z, X, M}^{-1}}{\longrightarrow} Z *(X * M)
$$

for all $X \in \mathscr{C}$ and $M \in \mathcal{M}$, and its left $\mathscr{C}_{\mathcal{M}}^{*}$-module functor structure

$$
F(Z * M) \stackrel{\simeq}{\rightarrow} Z * F(M)
$$

for $F \in \mathscr{C}_{\mathcal{M}}^{*}$ is given using the $\mathscr{b}$-module functor structure of $F$.

One defines a functor

$$
\tilde{a}_{\mathcal{M}}: \mathscr{L}\left(\mathscr{C}_{\mathcal{M}}^{*}\right) \stackrel{\sim}{\longrightarrow}\left(\mathscr{C} \nabla \mathscr{C}_{\mathcal{M}}^{*}\right)_{\mathcal{M}}^{*}
$$

in an analogous way.

The composition $\tilde{a}_{\mathcal{M}}^{-1} \circ a_{\mathcal{M}}$ is a braided tensor equivalence between $\mathscr{L}(\mathscr{C})$ and $\mathscr{L}\left(\mathscr{C}_{\mathcal{M}}^{*}\right)^{\mathrm{rev}}=\mathscr{L}\left(\left(\mathscr{C}_{\mathcal{M}}^{*}\right)^{\mathrm{op}}\right)$.

When $\mathcal{M}$ is an invertible $\mathscr{C}$-bimodule category, the composition of $\tilde{a}_{\mathcal{M}}$ and the braided tensor equivalence $\mathscr{L}(\mathscr{C}) \stackrel{\sim}{\rightarrow} \mathscr{L}\left(\left(\mathscr{C}_{\mathcal{M}}^{*}\right)^{\mathrm{op}}\right)$ induced by the tensor equivalence

$$
L: \mathscr{C} \stackrel{\sim}{\longrightarrow}\left(\mathscr{C}_{\mathcal{M}}^{*}\right)^{\mathrm{op}}: X \mapsto ? * X
$$

from Remark 2.11 gives a tensor equivalence

$$
b_{\mathcal{M}}: \mathscr{L}(\mathscr{C}) \stackrel{\sim}{\longrightarrow}\left(\mathscr{C} \nabla \mathscr{C}_{\mathcal{M}}^{*}\right)_{\mathcal{M}}^{*}:(Z, \gamma) \mapsto ? * Z,
$$

where the left $\mathscr{b}$-module functor structure of $b_{\mu}(Z, \gamma)$ is given by the middle associativity constraint of $M$,

$$
X *(M * Z) \stackrel{a_{X, M, Z}}{\longrightarrow}(X * M) * Z,
$$

while the right $\mathscr{C}$-module functor structure (which is the same as the left $C_{\mathcal{M}}^{*}$-module functor structure upon the identification $\left.\mathscr{C}_{\mathcal{M}}^{*} \simeq \mathscr{C}^{\text {op }}\right)$ of $b_{\mathcal{M}}(Z, \gamma)$ is given using the right $\mathscr{C}$-module associativity constraint of $\mathcal{M}$ and the half-braiding:

$$
(M * Z) * Y \stackrel{a_{M, Z, Y}}{\longrightarrow} M *(Z \otimes Y) \stackrel{\gamma_{Y}^{-1}}{\longrightarrow} M *(Y \otimes Z) \stackrel{a_{M, Y, Z}^{-1}}{\longrightarrow}(M * Y) * Z,
$$

for all $X, Y \in \mathscr{C}$ and $M \in \mathcal{M}$.

Thus, we have a canonical braided tensor autoequivalence

$$
\Phi(\mathcal{M})=b_{\mathcal{M}}^{-1} \circ a_{\mathcal{M}}: \mathscr{L}(\mathscr{C}) \rightarrow \mathscr{L}(\mathscr{C}) .
$$

The following result was proved in [Etingof et al. 2010, Section 5] when $\mathscr{b}$ is a fusion category. This argument carries over verbatim to the case of finite tensor categories. We recall the proof for the reader's convenience and also for future reference. 
Theorem 4.1. Let $\mathscr{C}$ be a finite tensor category. The assignment $M \mapsto \Phi(M)$, where $\Phi(\mathcal{M})$ is defined in (44), gives rise to a group isomorphism

$$
\Phi: \operatorname{BrPic}(\mathscr{C}) \stackrel{\simeq}{\rightarrow} \operatorname{Aut}^{\mathrm{br}}(\mathscr{L}(\mathscr{C})) .
$$

Proof. To see that $\Phi$ is a homomorphism observe that the $\mathscr{C}$-bimodule functor of right multiplication by an object $Z \in \mathscr{L}(\mathscr{C})$ on $M \nabla_{\mathscr{C}} \mathcal{N}$, where $M$ and $\mathcal{N}$ are invertible $\mathscr{C}$-bimodule categories, is isomorphic to the well-defined functor of "middle" multiplication by $(\Phi(\mathcal{N}))(Z)$, which, in turn, is isomorphic to the functor of left multiplication by $(\Phi(\mathcal{M}) \circ \Phi(\mathcal{N}))(Z)$. This gives a natural isomorphism of tensor functors $\Phi(\mathcal{M}) \circ \Phi(\mathcal{N}) \cong \Phi\left(\mathcal{M} \nabla_{\mathscr{C}} \mathcal{N}\right)$. Hence, $\Phi$ is a homomorphism.

Let us recall the construction of the map

$$
\Psi: \operatorname{Aut}^{\mathrm{br}}(\mathscr{L}(\mathscr{C})) \rightarrow \operatorname{BrPic}(\mathscr{C}),
$$

inverse to the homomorphism (45).

Let $F: \mathscr{L}(\mathscr{C}) \rightarrow \mathscr{C}$ and $I: \mathscr{C} \rightarrow \mathscr{L}(\mathscr{b})$ denote the canonical forgetful functor and its right adjoint. Given a braided autoequivalence $\alpha \in \operatorname{Aut}^{\mathrm{br}}(\mathscr{L}(\mathscr{C}))$ let $L_{\alpha}:=\alpha^{-1}(I(\mathbf{1}))$. The category ${ }_{L_{\alpha}} \mathscr{L}(\mathscr{C})$ is a finite tensor category with respect to $\otimes_{L_{\alpha}}$.

Let us show that the algebra $F\left(L_{\alpha}\right) \in \mathscr{C}$ is exact, that is, that the category $L_{\alpha} \mathscr{C}$ of $F\left(L_{\alpha}\right)$-modules in $\mathscr{C}$ is exact. By Lemma 3.2 this category is equivalent to $\operatorname{Fun}_{\mathscr{L}(\mathscr{C})}\left(\mathscr{L}(\mathscr{C})_{L_{\alpha}}, \mathscr{C}\right)$ as a $\mathscr{C}$-module category. By Remark 2.8 the latter category is exact as a Fun $\mathscr{L}_{(\mathscr{C})}(\mathscr{C}, \mathscr{C})$-module category. In particular, it is exact as a $\mathscr{C}$-module category.

Let

$$
F\left(L_{\alpha}\right)=\bigoplus_{i \in J} L_{\alpha}^{i}
$$

be the decomposition of $F\left(L_{\alpha}\right)$ into a direct sum of indecomposable exact algebras in $\mathscr{b}$.

For any $i \in J$ the composition

$$
\mathscr{C} \stackrel{\iota}{\rightarrow} L_{\alpha} \mathscr{L}(\mathscr{C}) \stackrel{F}{\rightarrow} F\left(L_{\alpha}\right) \mathscr{C}_{F\left(L_{\alpha}\right)} \stackrel{\pi_{i}}{\rightarrow} L_{\alpha}^{i} \mathscr{C}_{L_{\alpha}^{i}}
$$

is a tensor equivalence, where

$$
\iota: \mathscr{C} \stackrel{\sim}{\longrightarrow} L_{\alpha} \mathscr{L}(\mathscr{C}): X \mapsto \alpha^{-1}(I(X))
$$

and $\pi_{i}$ is a projection from $F\left(L_{\alpha}\right) \mathscr{C}_{F\left(L_{\alpha}\right)}=\bigoplus_{i, j \in J} L_{i} \mathscr{C}_{L_{j}}$ to the $(i, i)$ component.

Hence, $\mathscr{C}_{L_{i}}$ gets a structure of an invertible $\mathscr{C}$-bimodule category. Its equivalence class does not depend on a particular $i \in J$. One sets $\Psi(\alpha):=\mathscr{C}_{L_{i}}$.

The verification of the identities $\Phi \circ \Psi=\mathrm{id}$ and $\Psi \circ \Phi=\mathrm{id}$ is the same as in [Etingof et al. 2010, Section 5.3]. 
Remark 4.2. Note that $\operatorname{BrPic}(\mathscr{C})$ and $\operatorname{Aut}^{\text {br }}(\mathscr{L}(\mathscr{C}))$ are monoidal groupoids (that is, monoidal categories in which every object is invertible). In fact, the assignment (45) is a monoidal equivalence rather than just a group isomorphism, see [Etingof et al. 2010, Section 5].

4B. The image of Pic $(\mathscr{C})$ in $\operatorname{Aut}^{\mathrm{br}}(\mathscr{E}(\mathscr{C}))$. Recall from Section $2 \mathrm{H}$ that the group $\operatorname{BrPic}(\mathscr{C})$ contains a subgroup Pic $(\mathscr{C})$ consisting of equivalence classes of invertible $\mathscr{C}$-module categories (regarded as one-sided $\mathscr{C}$-bimodule categories).

Our goal now is to describe the image of $\operatorname{Pic}(\mathscr{C})$ in $\operatorname{Aut}^{\text {br }}(\mathscr{L}(\mathscr{C}))$ under isomorphism (45).

Let $\operatorname{Aut}^{\mathrm{br}}(\mathscr{L}(\mathscr{C}) ; \mathscr{C}) \subset \operatorname{Aut}^{\mathrm{br}}(\mathscr{L}(\mathscr{C}))$ be the subgroup consisting of isomorphism classes of braided autoequivalences of $\mathscr{E}(\mathscr{C})$ trivializable on $\mathscr{C}$, see Definition 2.1.

The next theorem was suggested to us by V. Drinfeld.

Theorem 4.3. Let $\mathscr{C}$ be a braided tensor category. The canonical isomorphism $\Phi: \operatorname{BrPic}(\mathscr{C}) \stackrel{\sim}{\rightarrow} \operatorname{Aut}^{\mathrm{br}}(\mathscr{L}(\mathscr{C}))$ restricts to an isomorphism

$$
\left.\Phi\right|_{\operatorname{Pic}(\mathscr{C})}: \operatorname{Pic}(\mathscr{C}) \stackrel{\simeq}{\rightarrow} \operatorname{Aut}^{\mathrm{br}}(\mathscr{L}(\mathscr{C}) ; \mathscr{C}) .
$$

Proof. First, let us show that $\Phi(\operatorname{Pic}(\mathscr{C})) \subset \operatorname{Aut}^{\mathrm{br}}(\mathscr{L}(\mathscr{C}) ; \mathscr{C})$. Let $\mathcal{M}$ be an invertible one-sided $\mathscr{C}$-module category. Let $\Phi(M) \in \operatorname{Aut}^{\mathrm{br}}(\mathscr{L}(\mathscr{C}))$ be the braided autoequivalence of $\mathscr{L}(\mathscr{C})$ defined in Section 4A. The equivalences $a_{\mathcal{M}}$ and $b_{\mathcal{M}}$ defined in (37) and (41) can be explicitly described as follows. Let $(Z, \gamma)$ be an object in $\mathscr{L}(\mathscr{C})$, where

$$
\gamma_{X}: X \otimes Z \rightarrow Z \otimes X, \quad X \in \mathscr{C}
$$

is the half-braiding. Then $a_{\mathcal{M}}(Z, \gamma)(M)=Z * M$ and its left and right $\mathscr{b}$-module functor structures are found by translating (38) and (39) to our setting:

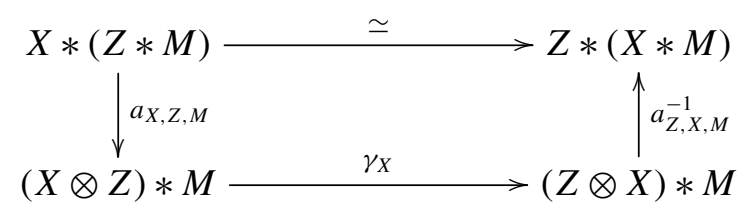

and

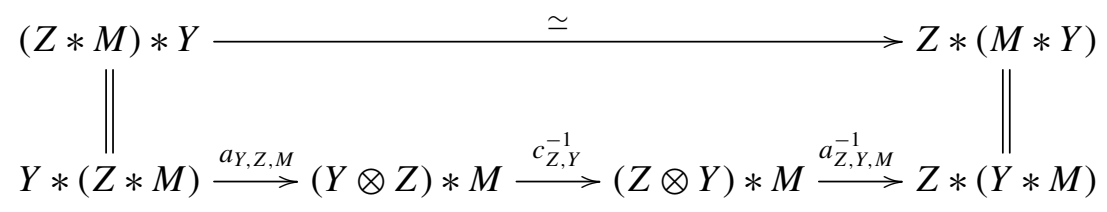

for all $X, Y \in \mathscr{C}$ and $M \in \mathcal{M}$, where $a$ denotes the left $\mathscr{b}$-module associativity constraint of $\mu$. 
Also, $b_{\mu}(Z, \gamma)(M)=M * Z=Z * M$ as a functor and its left and right $\mathscr{b}$-module functor structures are found from (42) and (43):

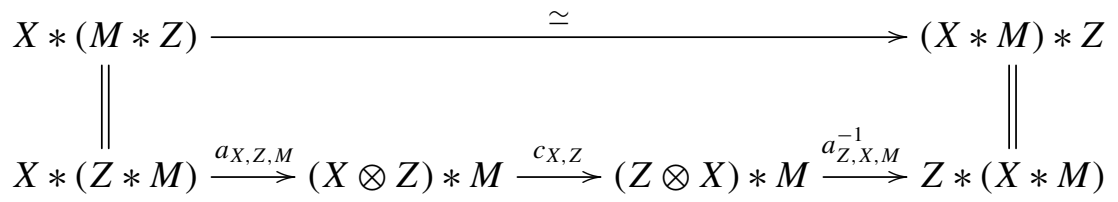

and

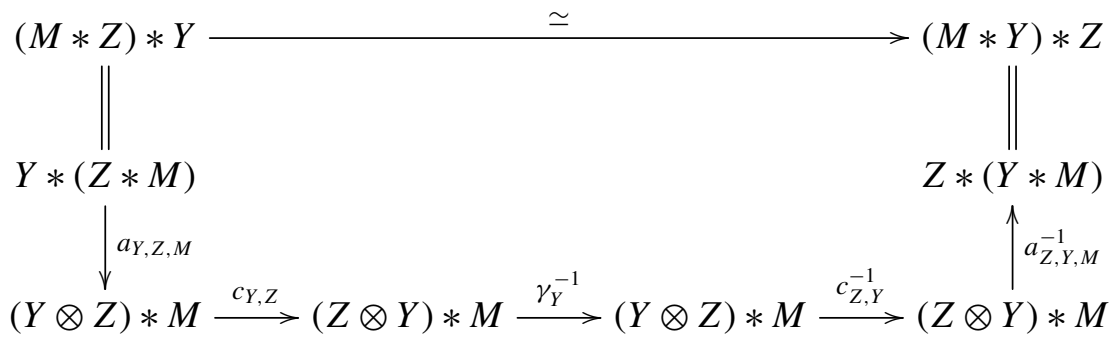

for all $X, Y \in \mathscr{C}$ and $M \in \mathcal{M}$.

The diagrams (52) and (51) are nothing but middle associativity isomorphism (17) and its inverse. The diagram (53) uses the right $\mathscr{C}$-module associativity (16) and its inverse as well as the half-braiding of $Z$.

Since $\mathscr{C}$ is embedded into $\mathscr{L}(\mathscr{C})$ via

$$
Z \mapsto\left(Z, c_{-, Z}\right),
$$

that is, $\gamma_{X}=c_{X, Z}$ in this case, we see from (50), (51) and (52), (53) that the restrictions of $a_{\mathcal{M}}$ and $b_{\mathcal{M}}$ on the subcategory $\mathscr{C} \subset \mathscr{L}(\mathscr{C})$ coincide, that is, $\Phi(\mathcal{M})$ is trivializable on $\mathscr{C}$. So $\Phi(\operatorname{Pic}(\mathscr{C})) \subset \operatorname{Aut}^{\text {br }}(\mathscr{L}(\mathscr{C}) ; \mathscr{C})$.

It remains to show that $\Phi(\operatorname{Pic}(\mathscr{C}))=\operatorname{Aut}^{\mathrm{br}}(\mathscr{L}(\mathscr{C}) ; \mathscr{C})$. Let $\alpha \in \operatorname{Aut}^{\mathrm{br}}(\mathscr{L}(\mathscr{C}) ; \mathscr{C})$. We need to show that the equivalence class of invertible $\mathscr{C}$-bimodule category $M:=\Psi(\alpha)$ (where $\Psi: \operatorname{BrPic}(\mathscr{C}) \rightarrow \operatorname{Aut}^{\text {br }}(\mathscr{L}(\mathscr{C}))$ is the inverse of $\Phi$, see (46)) is in $\operatorname{Pic}(\mathscr{C})$.

According to the description from the proof of Theorem $4.1 \mathcal{M}$ is equivalent to

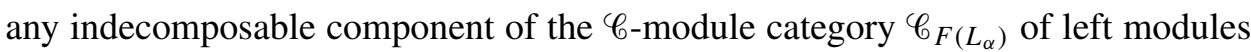
over the algebra $F\left(L_{\alpha}\right)$, where $L_{\alpha}=\alpha^{-1}(I(\mathbf{1})) \in \mathscr{L}(\mathscr{C})$. Thus, it suffices to show that the $\mathscr{C}$-bimodule category $\mathscr{C}_{F\left(L_{\alpha}\right)}$ is one-sided.

The left action of $X \in \mathscr{C}$ on $\mathscr{C}_{F\left(L_{\alpha}\right)}$ is via tensor multiplication:

$$
X * M=X \otimes M .
$$

The right action of $X$ is via module multiplication over $F\left(L_{\alpha}\right)$ with the image of $X$ under equivalence (47). Let us describe this action explicitly. Since $I(X) \cong X \otimes I(1)$ for all $X \in \mathscr{C} \subset \mathscr{L}(\mathscr{C})$ and $\alpha$ is trivializable on $\mathscr{C}$ we see that equivalence (48) in 
our situation becomes

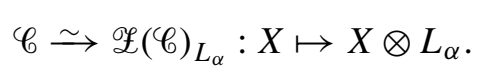

Therefore, the right action of $X$ on $\mathscr{C}_{F\left(L_{\alpha}\right)}$ is given by

$$
M * X=M \otimes_{F\left(L_{\alpha}\right)}\left(X \otimes F\left(L_{\alpha}\right)\right) \cong M \otimes X
$$

for all $X \in \mathscr{C}, M \in \mathscr{C}_{F\left(L_{\alpha}\right)}$. The action of $F\left(L_{\alpha}\right)$ on $M * X \cong M \otimes X$ is given by

$$
M \otimes X \otimes F\left(L_{\alpha}\right) \stackrel{1 \otimes c_{X, F\left(L_{\alpha}\right)}}{\longrightarrow} M \otimes F\left(L_{\alpha}\right) \otimes X \stackrel{\rho_{M} \otimes 1}{\longrightarrow} M \otimes X,
$$

where we omit the associativity constraints. Here $\rho_{M}: M \otimes F\left(L_{\alpha}\right) \rightarrow M$ denotes the $F\left(L_{\alpha}\right)$-module structure on $M$.

We have a natural family of $F\left(L_{\alpha}\right)$-module isomorphisms

$$
d_{M, X}:=c_{M, X}: M \otimes X \rightarrow X \otimes M .
$$

To show that the $\mathscr{b}$-bimodule category $\mathscr{C}_{F\left(L_{\alpha}\right)}$ is one-sided we need to check that isomorphisms $d_{X, M}$ satisfy commutative diagrams (20) and (21). But these diagrams are nothing but hexagon axioms of the braiding.

Thus, $\operatorname{Aut}^{\mathrm{br}}(\mathscr{L}(\mathscr{C}) ; \mathscr{C}) \subset \Phi(\operatorname{Pic}(\mathscr{C}))$ and the proof is complete.

4C. A characterization of the Picard crossed module. Let $\mathscr{b}$ be a finite braided tensor category. There is a canonical homomorphism

$$
\Sigma: \operatorname{Aut}^{\mathrm{br}}(\mathscr{L}(\mathscr{b}) ; \mathscr{C}) \rightarrow \operatorname{Aut}^{\mathrm{br}}(\mathscr{C})
$$

defined as follows. Every braided autoequivalence $\alpha \in \mathrm{Aut}^{\mathrm{br}}(\mathscr{L}(\mathscr{C}))$ trivializable on $\mathscr{C}$ maps the centralizer $\mathscr{C}$ in $\mathscr{L}(\mathscr{C})$ to itself. This centralizer is $\mathscr{C}^{\text {rev }} \subset \mathscr{L}(\mathscr{C})$. Hence, $\alpha$ restricts to a braided autoequivalence of $\mathscr{C}^{\text {rev }}$, that is, to an element of $\operatorname{Aut}^{\mathrm{br}}\left(\mathscr{C}^{\text {rev }}\right)=\operatorname{Aut}^{\mathrm{br}}(\mathscr{C})$ which we denote $\Sigma(\alpha)$.

Lemma 4.4. Let $\mathscr{C}$ be a braided tensor category. The composition

$$
\operatorname{Pic}(\mathscr{C}) \stackrel{\Phi}{\rightarrow} \operatorname{Aut}^{\mathrm{br}}(\mathscr{L}(\mathscr{C}) ; \mathscr{C}) \stackrel{\Sigma}{\rightarrow} \operatorname{Aut}^{\mathrm{br}}(\mathscr{C})
$$

coincides with homomorphism $\partial: \operatorname{Pic}(\mathscr{C}) \rightarrow \operatorname{Aut}^{\mathrm{br}}(\mathfrak{C})$ defined in (24).

Proof. We need to show that for each invertible $\mathscr{b}$-module category $\mathcal{M}$ the restriction of the braided autoequivalence $\Phi(\mathcal{M})$ on $\mathscr{C}^{\text {rev }} \subset \mathscr{L}(\mathscr{C})$ is isomorphic to $\partial_{\mathcal{M}}$ defined in (23). This result follows from comparing definitions. Indeed, $\Phi(\mathcal{M})=b_{\mathcal{M}}^{-1} \circ a_{\mathcal{M}}$, where $a_{\mathcal{M}}$ and $b_{\mathcal{M}}$ are defined in (37) and (41), and $\partial_{\mathcal{M}}=\left(\alpha_{\mathcal{M}}^{-}\right)^{-1} \circ \alpha_{\mathcal{M}}^{+}$, where $\alpha_{\mathcal{M}}^{ \pm}$ are defined in (22). 
Thus, it suffices to check the commutativity of the diagrams
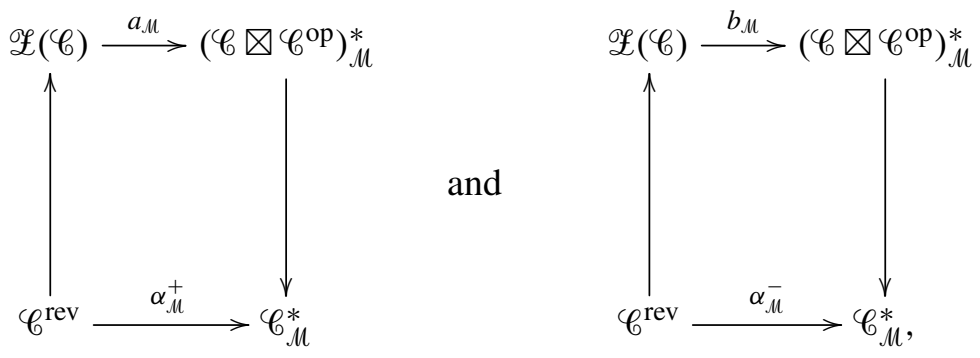

where the arrows $\mathscr{C}^{\text {rev }} \rightarrow \mathscr{L}(\mathscr{C})$ are given by the embedding (4) and the arrows $\left(\mathscr{C} \nabla \mathscr{C}^{\mathrm{op}}\right)_{\mathcal{M}}^{*} \rightarrow \mathscr{C}_{\mathcal{M}}^{*}$ are given by the restriction of $\mathscr{C}$-bimodule functors to left $\mathscr{C}$ module functors. The commutativity is checked directly using definitions of $\alpha_{M}^{ \pm}$in Section 2I and explicit formulas (50) and (52) for the $\mathscr{b}$-module functor structures of $a_{\mathcal{M}}(Z, \gamma)$ and $b_{\mu}(Z, \gamma)$, where $(Z, \gamma)$ is an object in $\mathscr{L}(\mathscr{C})$. In the bottom row of (58) we use that $\mathscr{C}^{\text {rev }}=\mathscr{C}$ as tensor categories.

Hence, $\left.\Phi(\mathcal{M})\right|_{\mathscr{C}^{\text {rev }}}=\partial_{\mathcal{M}}$ in $\operatorname{Aut}^{\mathrm{br}}(\mathscr{C})=\operatorname{Aut}^{\mathrm{br}}\left(\mathscr{C}^{\mathrm{rev}}\right)$.

Remark 4.5. Lemma 4.4 shows that the homomorphism $\partial: \operatorname{Pic}(\mathscr{C}) \rightarrow \operatorname{Aut}(\mathscr{C})$ defined in (24) factors through $\operatorname{Pic}(\mathscr{C}) \rightarrow \operatorname{Aut}^{\mathrm{br}}(\mathscr{C})$.

The next corollary was established in [Etingof et al. 2010] for braided fusion categories.

Corollary 4.6. Let $\mathscr{C}$ be a factorizable braided tensor category. Then $\partial: \operatorname{Pic}(\mathscr{C}) \rightarrow$ $\operatorname{Aut}^{\mathrm{br}}(\mathscr{C})$ is an isomorphism.

Proof. We have $\mathscr{L}(\mathscr{C}) \cong \mathscr{C} \otimes \mathscr{C}^{\text {rev }}$ and $\operatorname{Aut}^{\text {br }}(\mathscr{L}(\mathscr{C}) ; \mathscr{C})=\operatorname{Aut}^{\text {br }}\left(\mathscr{C}^{\text {rev }}\right)=\operatorname{Aut}^{\text {br }}(\mathscr{C})$.

There is canonical action of $\operatorname{Aut}^{\mathrm{br}}(\mathscr{C})$ on $\operatorname{Aut}^{\mathrm{br}}(\mathscr{L}(\mathscr{C}) ; \mathscr{C})$ defined as follows. Any tensor autoequivalence $g$ of $\mathscr{C}$ induces a braided autoequivalence $\tilde{g} \in \operatorname{Aut}^{\mathrm{br}}(\mathscr{E}(\mathscr{C}))$ :

$$
\tilde{g}(Z, \gamma)=\left(g(Z), \gamma^{g}\right),
$$

where $\left(\gamma^{g}\right)_{X}: X \otimes g(Z) \stackrel{\sim}{\rightarrow} g(Z) \otimes X$ is given by $\left(\gamma^{g}\right)_{X}=g\left(\gamma_{g^{-1}(X)}\right)$.

For all $g \in \operatorname{Aut}^{\mathrm{br}}(\mathscr{C})$ and $\alpha \in \operatorname{Aut}^{\mathrm{br}}(\mathscr{L}(\mathscr{C}) ; \mathscr{C})$ set

$$
{ }^{g} \alpha:=\tilde{g} \circ \alpha \circ \tilde{g}^{-1} .
$$

It is clear that ${ }^{g} \alpha$ is trivializable on $\mathscr{C}$, that is, (59) defines the required action.

Lemma 4.7. The isomorphism

$$
\Phi: \operatorname{Pic}(\mathscr{C}) \stackrel{\sim}{\longrightarrow} \operatorname{Aut}^{\mathrm{br}}(\mathscr{L}(\mathscr{C}) ; \mathscr{C})
$$

is $\mathrm{Aut}^{\mathrm{br}}(\mathscr{C})$-equivariant; that is,

$$
\Phi\left({ }^{g} \mathcal{M}\right)={ }^{g} \Phi(\mathcal{M})
$$


for all $g \in \operatorname{Aut}^{\mathrm{br}}(\mathscr{C})$ and $M \in \operatorname{Pic}(\mathscr{C})$.

Proof. This is an immediate consequence of the identities

$$
a_{g_{\mathcal{M}}}=a_{\mathcal{M}} \circ \tilde{g}^{-1} \quad \text { and } \quad b_{g_{\mathcal{M}}}=b_{\mathcal{M}} \circ \tilde{g}^{-1} \text {. }
$$

We have $\Phi\left({ }^{g} \mathcal{M}\right)=b_{g_{\mathcal{M}}}^{-1} \circ a_{g_{\mathcal{M}}}=\tilde{g} \circ \Phi(\mathcal{M}) \circ \tilde{g}^{-1}={ }^{g} \Phi(\mathcal{M})$.

Corollary 4.8. The pair of groups $\left(\operatorname{Aut}^{\mathrm{br}}(\mathscr{C})\right.$, $\left.\operatorname{Aut}^{\mathrm{br}}(\mathscr{L}(\mathscr{C}) ; \mathscr{C})\right)$ along with the action (59) and homomorphism $\Sigma: \mathrm{Aut}^{\mathrm{br}}(\mathscr{L}(\mathscr{C}) ; \mathscr{C}) \rightarrow \mathrm{Aut}^{\mathrm{br}}(\mathscr{C})$ from $(57)$ is a crossed module.

Proof. This follows from Lemmas 4.4 and 4.7.

We will call the crossed module $\left(\operatorname{Aut}^{\mathrm{br}}(\mathscr{C}), \operatorname{Aut}^{\mathrm{br}}(\mathscr{L}(\mathscr{C}) ; \mathscr{C})\right)$ the autoequivalence crossed module of $\mathscr{C}$ and denote it by $\mathfrak{A}(\mathscr{C})$.

Corollary 4.9. The pair of group isomorphisms $\left(\mathrm{id}_{\mathrm{Aut}^{\mathrm{br}}(\mathscr{C})}, \Phi\right)$ is an isomorphism of crossed modules

$$
\mathfrak{P}(\mathscr{C}) \cong \mathfrak{A}(\mathscr{C}) .
$$

Proof. This follows from Lemmas 4.4 and 4.7.

4D. On the kernel and cokernel of $\partial: \operatorname{Pic}(\mathscr{C}) \rightarrow \operatorname{Aut}^{\text {br }}(\mathscr{C})$. Since the Picard crossed module $\mathfrak{P}(\mathscr{C})$ is isomorphic to the autoequivalence crossed module of $\mathfrak{A}(\mathscr{C})$, the kernel of $\partial: \operatorname{Pic}(\mathscr{C}) \rightarrow \operatorname{Aut}^{\mathrm{br}}(\mathscr{C})$ is isomorphic to the kernel of the restriction map $\partial: \operatorname{Aut}^{\mathrm{br}}(\mathscr{L}(\mathscr{C}), \mathscr{C}) \rightarrow \operatorname{Aut}^{\mathrm{br}}(\mathscr{C})$.

The natural tensor embeddings $\mathscr{L}_{\text {sym }}(\mathscr{C}) \hookrightarrow \mathscr{C}, \mathscr{C}^{\text {rev }}$ allow us to look at $\mathscr{C}$ and $\mathscr{C}^{\text {rev }}$ as $\mathscr{L}_{\text {sym }}(\mathscr{C})$-module categories. The functor $\mathscr{C} \otimes \mathscr{C}^{\text {rev }} \rightarrow \mathscr{L}(\mathscr{C})$ of (5) is clearly balanced with respect to these module structures. Hence, it factors through $\mathscr{C} \nabla_{\mathscr{L}_{\mathrm{sym}}(\mathscr{C})} \mathscr{C}^{\text {rev }}$. Here the tensor product $\mathscr{C}_{\mathscr{L}_{\mathrm{sym}}(\mathscr{C})} \mathscr{C}^{\mathrm{rev}}$ of module categories over a symmetric tensor category $\mathscr{L}_{\text {sym }}(\mathscr{C})$ has a natural structure of braided tensor category, see [Davydov et al. 2013]. The image of $\mathscr{C}_{\mathscr{L}_{\text {sym }}(\mathscr{C})} \mathscr{C}^{\text {rev }}$ in $\mathscr{L}(\mathscr{C})$ coincides with the full tensor subcategory $\mathscr{C} \vee \mathscr{C}^{\text {rev }}$ generated by $\mathscr{C}$ and $\mathscr{C}^{\text {rev }}$ in $\mathscr{L}(\mathscr{C})$.

Proposition 4.10. The kernel of the restriction map $\partial: \operatorname{Aut}^{\mathrm{br}}(\mathscr{L}(\mathscr{C}), \mathscr{C}) \rightarrow \operatorname{Aut}^{\mathrm{br}}(\mathscr{C})$ coincides with the group $\mathrm{Aut}^{\mathrm{br}}\left(\mathscr{L}(\mathscr{C}), \mathscr{C} \vee \mathscr{C}^{\mathrm{rev}}\right)$ of braided autoequivalences of $\mathscr{L}(\mathscr{C})$ trivializable on $\mathscr{C} \vee \mathscr{C}^{\text {rev }}$.

Proof. The kernel of the restriction map $\partial: \operatorname{Aut}^{\mathrm{br}}(\mathscr{L}(\mathscr{C}), \mathscr{C}) \rightarrow \operatorname{Aut}^{\mathrm{br}}(\mathscr{C})$ coincides with the subgroup $\operatorname{Aut}^{\mathrm{br}}(\mathscr{L}(\mathscr{C}))$ of braided autoequivalences of $\mathscr{L}(\mathscr{C})$, trivializable on both $\mathscr{C}$ and $\mathscr{C}^{\text {rev }}$. All we need to show is that a braided autoequivalence of $\mathscr{L}(\mathscr{C})$ that is trivializable on both $\mathscr{C}$ and $\mathscr{C}^{\text {rev }}$ is trivializable on $\mathscr{C} \vee \mathscr{C}^{\text {rev }}$. 
A braided autoequivalence $F$ of $\mathscr{L}(\mathscr{C})$ stabilizing both $\mathscr{C}$ and $\mathscr{C}^{\text {rev }}$ and trivializable on $\mathscr{L}_{\text {sym }}(\mathscr{C})$ fits into a commutative diagram:

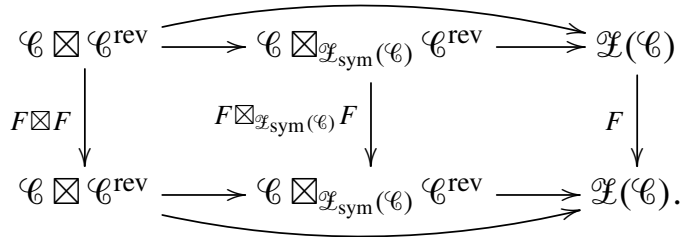

Thus a braided autoequivalence $F$ of $\mathscr{L}(\mathscr{C})$ that is trivializable on both $\mathscr{C}$ and $\mathscr{C}^{\text {rev }}$ is also trivializable on $\mathscr{C} \vee \mathscr{C}^{\text {rev }}$.

Note that there is a canonical homomorphism

$$
j: \operatorname{Pic}\left(\mathscr{Z}_{\mathrm{sym}}(\mathscr{C})\right) \rightarrow \operatorname{ker}\left(\operatorname{Pic}(\mathscr{C}) \stackrel{\partial}{\rightarrow} \operatorname{Aut}^{\mathrm{br}}\left(\mathscr{C} ; \mathscr{L}_{\mathrm{sym}}(\mathscr{C})\right)\right)
$$

given by the induction of module categories. Namely, if $\mathcal{M}$ is an invertible $\mathscr{L}_{\text {sym }}(\mathscr{C})$ module category then

$$
j(M)=\mathscr{C} \bigotimes_{\mathscr{L}_{\mathrm{sym}}(\mathscr{C})} \mathcal{M} .
$$

To see that $j(\mathcal{M})$ is in the kernel of $\partial$, let us take an algebra $A$ in $\mathscr{E}_{\text {sym }}(\mathscr{C})$ such that $\mathcal{M} \simeq \mathscr{Z}_{\text {sym }}(\mathscr{C})_{A}$. By Lemma 3.2 we have

$$
j(\mathcal{M})=\operatorname{Fun}_{\mathscr{L}_{\mathrm{sym}}(\mathscr{C})}(\mathscr{C}, \mathcal{M}) \simeq \mathscr{C}_{A} .
$$

The functors $\alpha_{j(\mathcal{M})}^{ \pm}$coincide with each other since $c_{X, A}=c_{A, X}^{-1}$ for all objects $X$ in $\mathscr{C}$, that is, $\partial(j(\mathcal{M}))$ is a trivial autoequivalence.

Let $\mathscr{F}_{\text {sym }}(\mathscr{C})$ be the symmetric center of $\mathscr{C}$, see Section $2 \mathrm{D}$. Clearly the restrictions of $\alpha_{\mathcal{M}}^{ \pm}$to $\mathscr{L}_{\text {sym }}(\mathscr{C})$ coincide. Hence for an invertible $\mathcal{M}$ the autoequivalence $\partial_{\mathcal{M}}$ is trivializable on $\mathscr{L}_{\text {sym }}(\mathscr{C})$, that is, the restriction of $\partial_{\mu}$ to $\mathscr{L}_{\text {sym }}(\mathscr{C})$ is isomorphic to the identity functor. Thus the homomorphism (24) factors as follows.

$$
\operatorname{Pic}(\mathscr{C}) \rightarrow \operatorname{Aut}^{\text {br }}\left(\mathscr{C} ; \mathscr{L}_{\text {sym }}(\mathscr{C})\right) \rightarrow \operatorname{Aut}^{\text {br }}(\mathscr{C})
$$

Hence, the restriction map defines canonical homomorphism from the cokernel of $\partial$ :

$$
\operatorname{coker}\left(\operatorname{Pic}(\mathscr{C}) \stackrel{\partial}{\rightarrow} \operatorname{Aut}^{\mathrm{br}}(\mathscr{C})\right) \rightarrow \operatorname{Aut}^{\mathrm{br}}\left(\mathscr{L}_{\mathrm{sym}}(\mathscr{C})\right)
$$

\section{The Picard crossed module of a pointed braided fusion category}

Let $A$ be a finite abelian group and let $q: A \rightarrow k^{\times}$be a quadratic form on $A$. In this section we explicitly compute the Picard crossed module of the pointed braided fusion category $\mathscr{b}:=\mathscr{C}(A, q)$ associated to the pair $(A, q)$ as in Example 2.2. 
Note that $\mathscr{C}(A, q)^{\mathrm{rev}} \simeq \mathscr{C}\left(A, q^{-1}\right)$.

5A. Invertible module categories over a braided pointed fusion category. The classification of module categories over pointed fusion categories is well-known [Ostrik 2003a]. Any indecomposable $\mathscr{C}$-module category $\mathcal{M}$ corresponds to a pair $(B, \gamma)$, where $B \subset A$ is a subgroup and $\gamma: B \times B \rightarrow k^{\times}$is a function such that

$$
d(\gamma)(x, y, z):=\frac{\gamma(x+y, z) \gamma(x, y)}{\gamma(x, y+z) \gamma(y, z)}=\omega(x, y, z), \quad x, y, z \in B .
$$

Here $\omega: A^{3} \rightarrow k^{\times}$is the 3 -cocycle defining the associativity constraint of $\mathscr{C}$.

The pair $(B, \gamma)$ is constructed from $M$ as follows. The simple objects of $M$ form a transitive $A$-set and $B$ denotes the stabilizer of a point in this set. The function $\gamma: B \times B \rightarrow k^{\times}$comes from the module associativity constraint of $\mu$. This function is determined by $M$ up to a 2-coboundary.

Let us define a function $\beta: B \times B \rightarrow k^{\times}$by

$$
\beta(x, y)=c(x, y) \frac{\gamma(x, y)}{\gamma(y, x)}, \quad x, y \in B
$$

where the function $c: A \times A \rightarrow k^{\times}$is defined in Example 2.2.

Proposition 5.1. The function (64) is bimultiplicative and satisfies

$$
\beta(x, x)=q(x) \text { for all } x \in B .
$$

Proof. For all $x, y, z \in B$ we compute

$$
\begin{aligned}
\beta(x, y+z) & \\
= & c(x, y+z) \frac{\gamma(x, y+z) \gamma(y, z)}{\gamma(y+z, x) \gamma(y, z)} \\
= & c(x, y+z) \frac{\gamma(x+y, z) \gamma(x, y)}{\gamma(y, z+x) \gamma(z, x)} \omega^{-1}(x, y, z) \omega^{-1}(y, z, x) \\
= & c(x, y+z) \frac{\gamma(y+x, z) \gamma(y, x)}{\gamma(y, x+z) \gamma(x, z)} \frac{\gamma(x, y)}{\gamma(y, x)} \frac{\gamma(x, z)}{\gamma(z, x)} \omega^{-1}(x, y, z) \omega^{-1}(y, z, x) \\
= & \beta(x, y) \beta(x, z) \frac{c(x, y+z)}{c(x, y) c(x, z)} \frac{\omega(y, x, z)}{\omega(x, y, z) \omega(y, z, x)} \\
& =\beta(x, y) \beta(x, z) .
\end{aligned}
$$

In the second and the fourth equalities we used identity (63) and in the last equality we used (7). Thus, $\beta$ is multiplicative in the second argument. That it is multiplicative in the first argument is proved in a similar way. Finally, the identity $\beta(x, x)=q(x)$ is obtained by setting $y=x$ in (64). 
Corollary 5.2. There is a bijection between

$$
\left\{\begin{array}{c}
\text { equivalence classes of } \\
\text { indecomposable module } \\
\mathscr{C}(A, q) \text {-categories }
\end{array}\right\} \text { and }\left\{\begin{array}{c}
\text { pairs }(B, \beta), \text { where } B \text { is a subgroup of } A, \\
\beta: B \times B \rightarrow k^{\times} \text {is bimultiplicative and } \\
\beta(x, x)=q(x), x \in B
\end{array}\right\} \text {. }
$$

Proof. Let $B$ be a subgroup of $A$ corresponding to an indecomposable $\mathscr{b}$-module category. Formula (64) defines a map between sets

$$
\left\{\begin{array}{c}
\text { maps } \gamma: B \times B \rightarrow k^{\times} \\
\text {such that } d(\gamma)=\omega \\
\text { modulo coboundaries }
\end{array}\right\} \rightarrow\left\{\begin{array}{c}
\beta \in \operatorname{Hom}\left(B^{\otimes 2}, k^{\times}\right) \\
\text {such that } \\
\beta(x, x)=q(x), x \in B
\end{array}\right\} .
$$

We need to prove that (66) is a bijection.

Let $\gamma_{1}, \gamma_{2}$ be 2-cochains on $B$ such that $d\left(\gamma_{1}\right)=d\left(\gamma_{2}\right)=\omega$ and such that

$$
c(x, y) \frac{\gamma_{1}(x, y)}{\gamma_{1}(y, x)}=c(x, y) \frac{\gamma_{2}(x, y)}{\gamma_{2}(y, x)}, \quad x, y \in B .
$$

Then $\gamma_{2} / \gamma_{1}$ is a symmetric 2-cocycle on $B$. Since a symmetric 2-cocycle is cohomologically trivial, $\gamma_{1}$ and $\gamma_{2}$ differ by a coboundary. Thus the map (66) is injective.

Consider the diagram

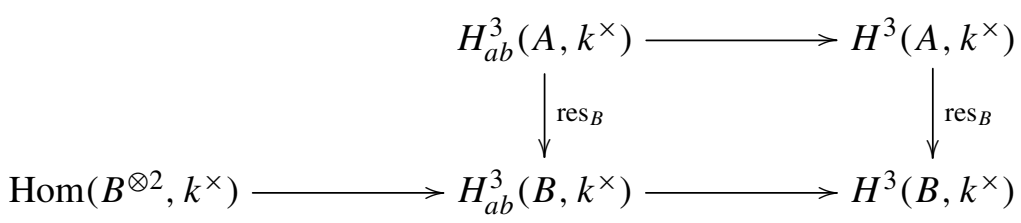

with commutative square and the bottom row exact in the middle term. (Abelian cohomology groups were defined in Example 2.2.) Let $q$ be a quadratic form on $A$, identified with an element of $H_{a b}^{3}\left(A, k^{\times}\right)$. It follows from diagram (67) that $q$ is in the kernel of the composition

$$
H_{a b}^{3}\left(A, k^{\times}\right) \rightarrow H^{3}\left(A, k^{\times}\right) \rightarrow H^{3}\left(B, k^{\times}\right)
$$

if and only if the restriction of $q$ to $B$ can be represented by some bimultiplicative $\beta: B^{\otimes 2} \rightarrow k^{\times}$. This proves surjectivity of (66).

Remark 5.3. Note that the condition (65) along with identity (12) imply

$$
\beta(x, y) \beta(y, x)=\sigma(x, y), \quad x, y \in B .
$$

By $\mathcal{M}(B, \beta)$ we will denote a module category corresponding to the pair $(B, \beta)$ under the bijection from Corollary 5.2.

The following lemma is a special case of the result proved in [Naidu 2007]: 
Lemma 5.4. Let $\mathcal{M}=\mathcal{M}(B, \beta)$ be a $\mathscr{C}(A, q)$-module category. Then the group $\operatorname{Aut}_{\mathscr{C}}(\mathcal{M})$ of isomorphism classes of $\mathscr{b}$-module autoequivalences of $\mathcal{M}$ fits into a short exact sequence:

$$
1 \longrightarrow \hat{B} \longrightarrow \operatorname{Aut}_{\mathscr{C}}(M) \longrightarrow A / B \longrightarrow 1
$$

Proof. The homomorphism $\operatorname{Aut}_{\mathscr{C}}(\mathcal{M}) \rightarrow A / B$ assigns the effect of a $\mathscr{C}$-equivalence on the set $A / B$ of simple objects of $\mathcal{M}$. It is clear that this homomorphism is surjective (it is enough to look at the images of $\alpha$-inductions).

The kernel of the homomorphism $\operatorname{Aut}_{\mathscr{C}}(\mathcal{M}) \rightarrow A / B$ consists of isomorphism classes of $\mathscr{C}$-equivalences isomorphic to the identity functor. With a choice of simple object $m \in \mathcal{M}$ a $\mathscr{b}$-module structure on the identity functor on $\mathcal{M}$ gives rise to a character $\psi \in \hat{B}$

$$
\psi(b) \operatorname{id}_{m}: m=b * m \rightarrow b * m=m .
$$

It follows from Shapiro's lemma that the character determines the $\mathscr{b}$-module structure.

Proposition 5.5. The $\mathscr{C}(A, q)$-module category $\mathcal{M}(B, \beta)$ is invertible if and only if the form $\beta: B \times B \rightarrow k^{\times}$is nondegenerate.

Proof. Note that $M=M(B, \beta)$ is invertible if and only if the $\alpha$-inductions

$$
\alpha_{\mathcal{M}}^{ \pm}: \mathscr{b} \rightarrow \operatorname{End}_{\mathscr{C}}(\mathcal{M})
$$

from Section 2I induce isomorphisms of groups $A \rightarrow \operatorname{Aut}_{\mathscr{C}}(M)$ on the level of isomorphism classes of objects. We can see that $\alpha$-inductions give morphisms of short exact sequences:

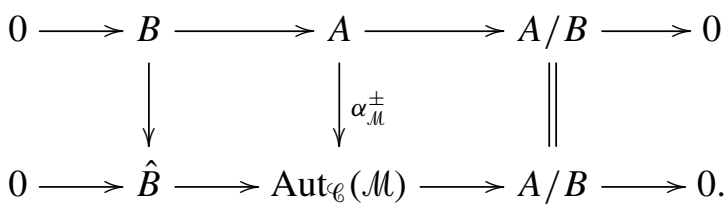

The homomorphisms $B \rightarrow \hat{B}$ can be recovered from the $\mathscr{C}$-module functor structures of $\alpha_{\mathcal{M}}^{ \pm}(a)$ for $a \in A$. The $\mathscr{b}$-module functor structure for $\alpha_{\mathcal{M}}^{+}(a)$ is given by the diagram

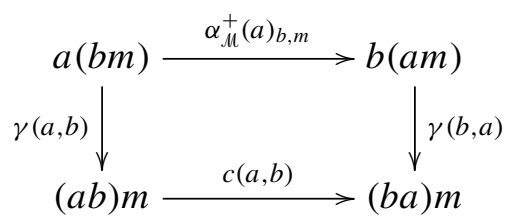

so that $\alpha_{\mathcal{M}}^{+}(a)_{b, m}=\beta(a, b)$ for $a, b \in B$. Here $m$ is a simple object of $\mathcal{M}$. Thus, the corresponding homomorphism $B \rightarrow \hat{B}$ has the form $b \rightarrow \beta(b,-)$. 
Similarly, the $\mathscr{b}$-structure for $\alpha_{\mathcal{M}}^{-}(a)$ is defined by

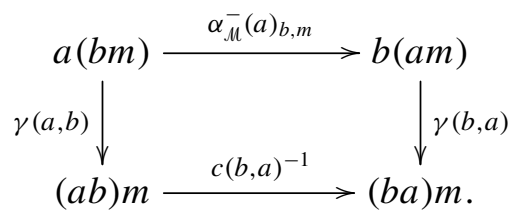

Hence, $\alpha_{\mu}^{-}(a)_{b, m}=\beta(b, a)^{-1}$ for $a, b \in B$ and the corresponding homomorphism $B \rightarrow \hat{B}$ has a form $b \rightarrow \beta(-, b)^{-1}$.

From the proof of Proposition 5.5 we have the following:

Corollary 5.6. The homomorphism $\partial: \operatorname{Pic}(\mathscr{C}(A, q)) \rightarrow \operatorname{Aut}^{\mathrm{br}}(A, q)$ sends the class of $\mathcal{M}(B, \beta)$ into the unique automorphism $g \in O(A, q)$ such that

- $g(B) \subset B$,

- $g$ induces the identity on $A / B$, and

- $\beta(b, g(c))=\beta(c, b)^{-1}$ for all $b, c \in B$.

Remark 5.7. It follows from (68) that the last condition in Corollary 5.6 can be written as $\beta(b, g(c)-c)=\sigma(b, c)^{-1}$ for all $b, c \in B$. This gives an alternative description of $g$ (compare [Davydov et al. 2011]; the graph of $-g$ is the Lagrangian subgroup $\Gamma(B, \beta) \subset\left(A \oplus A, q \oplus q^{-1}\right)$ there):

- $g(a)-a \in B$ for any $a \in A$ and

- $\beta(b, g(a)-a)=\sigma(b, a)^{-1}$ for all $b \in B$.

In accordance with the crossed module axiom (36) the map

$$
\partial: \operatorname{Pic}(\mathscr{C}(A, q)) \rightarrow O(A, q)
$$

is $O(A, q)$-equivariant: $\partial(h(B, \beta))=h \circ \partial(B, \beta) \circ h^{-1}$ for $h \in O(A, q)$. Here $h(B, \beta)=(h(B), h(\beta))$ with $h(\beta)(a, b)=\beta\left(h^{-1}(a), h^{-1}(b)\right)$ and ${ }^{h} M(B, \beta) \simeq$ $\mu(h(B, \beta))$.

This gives a description of the map $\partial$ for the Picard crossed module $\mathfrak{P}(\mathscr{C}(A, q))$. The part which is unclear in this presentation is the group structure of $\operatorname{Pic}(\mathscr{C}(A, q))$. It appears that the group operation is more accessible on the level of the autoequivalence crossed module $\mathfrak{A}(\mathscr{C}(A, q))$ (recall that $\mathfrak{A}(\mathscr{C}(A, q)) \simeq \mathfrak{P}(\mathscr{C}(A, q))$ by Corollary 4.9$)$. In the remaining sections we compute this crossed module.

5B. The center of a pointed braided fusion category. Let $\mathscr{C}=\mathscr{C}(A, q)$ be a pointed braided fusion category. The following fact is no doubt known to experts but we were unable to locate a reference in the literature:

Proposition 5.8. The center $\mathscr{L}(\mathscr{C})$ is pointed and $\mathscr{E}(\mathscr{C}) \simeq \mathscr{C}(A \oplus \hat{A}, Q)$, where

$$
Q(a, \phi)=\langle\phi, a\rangle q(a) \text {. }
$$


Proof. For any $a \in A$ and $\phi \in \hat{A}$ there is an invertible object $Z_{a, \phi}$ in $\mathscr{L}(\mathscr{C})$ which is equal to $a$ as an object of $\mathscr{C}$ and has a half-braiding given by

$$
c(x, a)\langle\phi, x\rangle \operatorname{id}_{a+x}: x \otimes Z_{a, \phi} \stackrel{\simeq}{\rightarrow} Z_{a, \phi} \otimes x,
$$

where $c: A^{\times 2} \rightarrow k^{\times}$is the function (6) determining the braiding of $\mathscr{b}$. That the morphism (70) is indeed a central structure on $a$ (that is, satisfies necessary coherence conditions) follows from identities (7) and (8).

Thus, $\mathscr{L}(\mathscr{C})$ contains $|A|^{2}$ nonisomorphic invertible simple objects. Since the dimension of $\mathscr{L}(\mathscr{C})$ is $\operatorname{dim}(\mathscr{C})^{2}=|A|^{2}$, the category $\mathscr{L}(\mathscr{C})$ is pointed. Furthermore, $Z_{a, \phi} \otimes Z_{a^{\prime}, \phi^{\prime}}=Z_{a a^{\prime}, \phi \phi^{\prime}}, a, a^{\prime} \in A, \phi, \phi^{\prime} \in \hat{A}$, that is, the group of invertible objects of $\mathscr{L}(\mathscr{C})$ is $A \oplus \hat{A}$. Finally, from (70) we see that the braiding on $Z_{a, \phi} \otimes Z_{a, \phi}$ is given by the scalar $\langle\phi, a\rangle q(a)$.

Remark 5.9. Let

$$
\sigma(a, b):=\frac{q(a+b)}{q(a) q(b)}, \quad x, y \in A
$$

be the bimultiplicative form corresponding to the quadratic form $q: A \rightarrow k^{\times}$. Then the bimultiplicative form corresponding to the form $Q$ defined in (69) is

$$
\begin{aligned}
B\left((a, \phi),\left(a^{\prime}, \phi^{\prime}\right)\right) & =\frac{Q\left(a+a^{\prime}, \phi+\phi^{\prime}\right)}{Q(a, \phi) Q\left(a^{\prime}, \phi^{\prime}\right)} \\
& =\left\langle\phi^{\prime}, a\right\rangle\left\langle\phi, a^{\prime}\right\rangle \sigma\left(a, a^{\prime}\right), \quad a, a^{\prime} \in A, \phi, \phi^{\prime} \in \hat{A} .
\end{aligned}
$$

Remark 5.10. Note that in general the category $\mathscr{L}\left(\operatorname{Vec}_{A}^{\omega}\right)$, where $A$ is an abelian group and $\omega \in Z^{3}\left(A, k^{\times}\right)$, is not pointed, see [Goff et al. 2007].

Let $\sigma: A \times A \rightarrow k^{\times}$be the symmetric bimultiplicative form (71). For any $a \in A$ define a homomorphism $\tilde{\sigma}: A \rightarrow \hat{A}$ by

$$
\langle\tilde{\sigma}(a), x\rangle=\sigma(a, x) \text { for all } x \in A .
$$

The embeddings $\mathscr{C}(A, q), \mathscr{C}(A, q)^{\mathrm{rev}} \hookrightarrow \mathscr{L}(\mathscr{C}(A, q))$ defined in (4) are given by injective orthogonal homomorphisms

$$
\begin{aligned}
(A, q) & \rightarrow(A \oplus \hat{A}, Q): a \mapsto(a, 0), \\
\left(A, q^{-1}\right) & \rightarrow(A \oplus \hat{A}, Q): a \mapsto(a,-\tilde{\sigma}(a)) .
\end{aligned}
$$

5C. The Picard group of $\mathscr{C}(\boldsymbol{A}, \boldsymbol{q})$. By Theorem 4.3 any invertible $\mathscr{C}(A, q)$-module category corresponds to an orthogonal automorphism $\alpha \in O(A \oplus \hat{A}, Q)$ such that $\alpha(a, 0)=(a, 0)$ for all $a \in A$.

Proposition 5.11. Let $f: \hat{A} \rightarrow$ A be a group homomorphism satisfying the following conditions:

(i) $\operatorname{id}_{\hat{A}}-\tilde{\sigma} f$ is invertible; 
(ii) $\langle\phi, f(\phi)\rangle=q(f(\phi))$ for all $\phi \in \hat{A}$.

Then the map

$$
\alpha_{f}(a, \phi)=(a+f(\phi), \phi-\tilde{\sigma} f(\phi)), \quad a \in A, \phi \in \hat{A}
$$

is an orthogonal automorphism of $(A \oplus \hat{A}, Q)$ that restricts to the identity on $A$.

Conversely, any orthogonal automorphism with this property is of the form (72) for a unique homomorphism $f: \hat{A} \rightarrow$ A satisfying conditions $(i)$ and (ii).

Proof. Suppose a group homomorphism $f: \hat{A} \rightarrow A$ is given. Clearly, $\alpha_{f}$ is a homomorphism and its restriction to $A$ is the identity. Condition (i) in the statement of the proposition is equivalent to $\alpha_{f}$ being invertible. Let us explore the property of $\alpha_{f}$ being orthogonal. We compute

$$
\begin{aligned}
Q\left(\alpha_{f}(a, \phi)\right) & =Q(a+f(\phi), \phi-\tilde{\sigma} f(\phi)) \\
& =Q(a, \phi) Q(f(\phi),-\tilde{\sigma} f(\phi)) B((a, \phi),(f(\phi),-\tilde{\sigma} f(\phi))) \\
& =Q(a, \phi) \sigma(f(\phi), f(\phi))^{-1} q(f(\phi))\langle\phi, f(\phi)\rangle \\
& =Q(a, \phi) q(f(\phi))^{-1}\langle\phi, f(\phi)\rangle,
\end{aligned}
$$

whence $\alpha_{f}$ is orthogonal if and only if condition (ii) is satisfied.

Let us prove the converse statement. Let $\alpha \in O(A \oplus \hat{A}, Q)$ be such that $\alpha$ restricts to the identity on $A$. Let $f: \hat{A} \rightarrow A$ and $g: \hat{A} \rightarrow \hat{A}$ be homomorphisms such that $\alpha(0, \phi)=(f(\phi), g(\phi))$ for all $\phi \in \hat{A}$. Since $\alpha$ preserves the quadratic form $Q$, the condition $Q(0, \phi)=1$ implies $Q(f(\phi), g(\phi))=1$, which is equivalent to

$$
\langle g(\phi), f(\phi)\rangle q(f(\phi))=1 .
$$

Next, for arbitrary $a \in A$ and $\phi \in \hat{A}$ we have

$$
\alpha(a, \phi)=(a+f(\phi), g(\phi)) .
$$

We have $Q(\alpha(a, \phi))=Q(a, \phi)=\langle\phi, a\rangle q(a)$. On the other hand, we compute

$$
\begin{aligned}
Q(\alpha(a, \phi)) & =Q(a+f(\phi), g(\phi)) \\
& =Q(a, 1) Q(f(\phi), g(\phi))\langle g(\phi), a\rangle \sigma(a, f(\phi)) \\
& =q(a)\langle g(\phi), a\rangle \sigma(f(\phi), a) \\
& =q(a)\langle g(\phi)+\tilde{\sigma} f(\phi), a\rangle .
\end{aligned}
$$

Comparing two expressions we obtain

$$
g(\phi)=\phi-\tilde{\sigma} f(\phi) \quad \text { for all } \phi \in \hat{A} .
$$

This along with (74) yields (72). 
Substituting (75) into (73) we obtain

$$
\langle\phi, f(\phi)\rangle q(f(\phi))=\langle\tilde{\sigma} f(\phi), f(\phi)\rangle=\sigma(f(\phi), f(\phi))=q(f(\phi))^{2},
$$

whence $\langle\phi, f(\phi)\rangle=q(f(\phi))$ as required.

Let $P(A, q)$ be the set of group homomorphisms $f: \hat{A} \rightarrow A$ satisfying conditions (i) and (ii) of Proposition 5.11, that is,

$$
P(A, q):=\left\{\begin{array}{c}
\text { homomorphisms } f: \hat{A} \rightarrow A \text { such that } \\
\operatorname{id}_{\hat{A}}-\tilde{\sigma} \circ f \text { is invertible and } \\
\langle\phi, f(\phi)\rangle=q(f(\phi)) \text { for all } \phi \in \hat{A}
\end{array}\right\} .
$$

Endow the set $P(A, q)$ with the binary operation

$$
f \diamond g=f+g-f \circ \tilde{\sigma} \circ g, \quad f, g \in P(A, q) .
$$

Proposition 5.12. The set $P(A, q)$ with the operation $\diamond$ defined in (77) is a group. Furthermore, the map

$$
f \mapsto \alpha_{f}: P(A, q) \rightarrow \operatorname{Aut}^{\mathrm{br}}(\mathscr{L}(\mathscr{C}(A, q)), \mathscr{C}(A, q)),
$$

where $\alpha_{f} \in \operatorname{Aut}^{\mathrm{br}}(\mathscr{L}(\mathscr{C}(A, q)))$ is defined in (72), is a group isomorphism.

Proof. By Proposition 5.11 the assignment (78) is a bijection. Since

$$
\alpha_{f} \circ \alpha_{g}=\alpha_{f \diamond g} \quad \text { for all } f, g \in P(A, q),
$$

we see that $P(A, q)$ is a group and the assignment (78) is a group isomorphism.

Remark 5.13. Clearly, the identity element of $P(A, q)$ is the zero homomorphism. Let us describe the inverse of $f \in P(A, q)$.

It is immediate from (77) that the inverse of $f$ is given by the formula

$$
f^{-1}=\left(f \circ \tilde{\sigma}-\mathrm{id}_{A}\right)^{-1} \circ f .
$$

Let $f^{*}: \hat{A} \rightarrow A$ denote the homomorphism dual to $f$. We claim that $f^{*} \in P(A, q)$ and that $f^{*}$ is the inverse of $f$ with respect to the multiplication $\diamond$. Indeed, equality of quadratic forms in condition (ii) of Proposition 5.11 implies equality of the corresponding bilinear forms

$$
\left\langle f+f^{*}(\phi), \psi\right\rangle=\sigma(f(\phi), f(\psi)), \quad \phi, \psi \in \hat{A},
$$

whence $f+f^{*}=f^{*} \circ \tilde{\sigma} \circ f$, that is, $f^{*}$ coincides with the right hand side of (79).

Corollary 5.14. There is a group isomorphism $P(A, q) \cong \operatorname{Pic}(\mathscr{C}(A, q))$.

Proof. This follows from Proposition 5.12 and Theorem 4.3. 
Remark 5.15. We have two parametrizations for the group $\operatorname{Pic}(\mathscr{C}(A, q))$. The first one is given in terms of pairs $(B, \beta)$, where $B \subset A$ is a subgroup and $\beta: B \times B \rightarrow k^{\times}$ is a nondegenerate bimultiplicative map such that $\beta(x, x)=q(x)$ for all $x \in B$, see Corollary 5.2 and Proposition 5.5. The second one is given in terms of the set $P(A, q)$ consisting of homomorphisms $f: \hat{A} \rightarrow A$ satisfying conditions listed in (76).

Let us establish a bijection between these parametrizations. Let $M=M(B, \beta)$ denote the invertible $\mathscr{C}(A, q)$-module category corresponding to a pair $(B, \beta)$ as above. Let $\Phi(M)$ denote the corresponding braided autoequivalence of $\mathscr{L}(\mathscr{C}(A, q)$ defined as in (44). By Proposition 5.11 $\Phi(\mathcal{M})=\alpha_{f}$ for a unique $f \in P(A, q)$. Let $\phi \in \hat{A}$ and let $b=f(\phi)$. Then $b$ is uniquely determined by the condition

$$
\Phi(M)\left(Z_{0, \phi}\right)=Z_{b, \psi} \quad \text { for some } \psi \in \hat{A} .
$$

Equivalently,

$$
a_{\mu}\left(Z_{0, \phi}\right)=b_{\mu}\left(Z_{b, \psi}\right),
$$

where $a_{\mu}$ and $b_{\mu}$ are functors defined in (37) and (41). Note that $b \in B$ since the functor $a_{\mathcal{M}}\left(Z_{0, \phi}\right)$ is identical on the classes of isomorphic objects of $\mathcal{M}$.

Take $x \in B$ and compare isomorphisms

$$
x \otimes a_{\mathcal{M}}\left(Z_{0, \phi}\right)(?) \stackrel{\simeq}{\rightarrow} a_{\mathcal{M}}\left(Z_{0, \phi}\right)(x \otimes ?)
$$

and

$$
x \otimes b_{\mathcal{M}}\left(Z_{b, \psi}\right)(?) \stackrel{\simeq}{\rightarrow} b_{\mathcal{M}}\left(Z_{b, \psi}\right)(x \otimes ?)
$$

coming from left $\mathscr{C}(A, q)$-module functor structures of $a_{\mathcal{M}}\left(Z_{0, \phi}\right)$ and $b_{\mathcal{M}}\left(Z_{0, \phi}\right)$.

Using Equations (50) and (70) we see that the isomorphism (80) is given by

$$
x \otimes\left(Z_{0, \phi} \otimes ?\right) \stackrel{\langle\phi, x\rangle}{\longrightarrow} Z_{0, \phi} \otimes(x \otimes ?) .
$$

On the other hand, using (52) we see that the isomorphism (81) is given by

$$
\begin{aligned}
x \otimes\left(Z_{b, \psi} \otimes ?\right) \stackrel{\gamma(x, b)}{\longrightarrow}\left(x \otimes Z_{b, \psi}\right) \otimes ? \\
\quad \stackrel{c(x, b)}{\longrightarrow}\left(Z_{b, \psi} \otimes x\right) \otimes ? \stackrel{\gamma(b, x)^{-1}}{\longrightarrow} Z_{b, \psi} \otimes(x \otimes ?),
\end{aligned}
$$

where $\gamma: B \times B \rightarrow k^{\times}$is the function that determines the module associativity of $M(B, \beta)$ - see (63) - and $c: A \times A \rightarrow k^{\times}$is the braiding of $\mathscr{C}(A, q)$. From (64) we see that the product of scalars in the composition (83) is equal to $\beta(x, b)$. Since $\beta$ is nondegenerate it follows that $b=f(\phi)$ is completely determined by the condition

$$
\langle\phi, x\rangle=\beta(x, b) .
$$


Thus, the homomorphism $f: \hat{A} \rightarrow A$ corresponding to $(B, \beta)$ is given by the composition

$$
f: \hat{A} \rightarrow \hat{B} \stackrel{\hat{\beta}}{\rightarrow} B \hookrightarrow A,
$$

where $\hat{A} \rightarrow \hat{B}$ is the surjection dual to the embedding $B \hookrightarrow A$ and $\hat{\beta}: \hat{B} \stackrel{\sim}{\longrightarrow} B$ is the isomorphism induced by $\beta$.

Example 5.16. (i) Suppose $q$ is nondegenerate (that is, the category $\mathscr{C}(A, q)$ is nondegenerate). Then $\tilde{\sigma}: A \rightarrow \hat{A}$ is an isomorphism and the map

$$
P(A, q) \rightarrow O(A, q) \quad \text { given by } f \mapsto \mathrm{id}_{A}-f \circ \tilde{\sigma}
$$

is an isomorphism.

(ii) Suppose $q=1$ (that is, the category $\mathscr{C}(A, q)$ is tannakian). Then

$$
P(A, q)=\{\phi: \hat{A} \rightarrow A \mid\langle\phi, f(\phi)\rangle=1\} .
$$

Thus, elements of $P(A, q)$ are identified with alternating bimultiplicative maps $\hat{A} \times \hat{A} \rightarrow k^{\times}$and

$$
P(A, q) \cong \bigwedge^{2} A \cong H^{2}\left(\hat{A}, k^{\times}\right) ;
$$

see [Etingof et al. 2010, Corollary 3.17].

(iii) Suppose that $\sigma=1$ but $q \neq 1$ (that is, the category $\mathscr{C}(A, q)$ is symmetric but not tannakian). In this case $q \in \hat{A}$ is a character of order 2 . Let $\langle q\rangle$ denote the subgroup of $\hat{A}$ generated by $q$. We have

$$
P(A, q) \cong \begin{cases}H^{2}\left(\hat{A}, k^{\times}\right) & \text {if }\langle q\rangle \text { is a direct summand in } \hat{A}, \\ H^{2}\left(\hat{A}, k^{\times}\right) \times \mathbb{Z} / 2 \mathbb{Z} & \text { otherwise. }\end{cases}
$$

This agrees with the result of [Carnovale 2006] in the case of semisimple Hopf algebras.

5D. Description of the Picard crossed module of $\mathscr{C}(A, q)$. Let $\mathscr{C}(A, q)$ be a pointed braided fusion category. By Corollary 4.9 the Picard crossed module of $\mathscr{C}$ is isomorphic to the autoequivalence crossed module $\mathfrak{A}(\mathscr{C}(A, q))$

$$
=\left(\operatorname{Aut}^{\mathrm{br}}(\mathscr{L}(\mathscr{C}(A, q)) ; \mathscr{C}(A, q)), \operatorname{Aut}^{\mathrm{br}}(\mathscr{C}(A, q))\right) \cong(P(A, q), O(A, q))
$$

introduced in Section 3D.

By Lemma 4.4 the structural homomorphism

$$
\partial: \operatorname{Pic}(\mathscr{C}(A, q)) \cong \operatorname{Aut}^{\mathrm{br}}(\mathscr{L}(\mathscr{C}(A, q)) ; \mathscr{C}(A, q)) \rightarrow \operatorname{Aut}^{\mathrm{br}}(\mathscr{C}(A, q))
$$

is given by restriction of the autoequivalences in $\operatorname{Aut}^{\mathrm{br}}(\mathscr{L}(\mathscr{C}(A, q)) ; \mathscr{C}(A, q))$ to $\mathscr{C}(A, q)^{\mathrm{rev}} \subset \mathscr{L}(\mathscr{C}(A, q))$. 
Let us describe $\partial$ explicitly. We already observed that the tensor subcategory $\mathscr{C}(A, q)^{\mathrm{rev}} \subset \mathscr{L}(\mathscr{C}(A, q))$ corresponds to the subgroup $\{(a,-\hat{a}) \mid a \in A\} \subset A \oplus \hat{A}$. Given $f \in P(A, q)$ we have

$$
\alpha_{f}(a,-\tilde{\sigma}(a))=(a-f \tilde{\sigma}(a),-(\tilde{\sigma}(a)-\tilde{\sigma} f \tilde{\sigma}(a)) .
$$

Hence,

$$
\partial(f)=\mathrm{id}_{A}-f \circ \tilde{\sigma}, \quad f \in P(A, q) .
$$

Next, for any $g \in O(A, q)$ let $\tilde{g} \in O(A \oplus \hat{A}, Q)$ be the orthogonal automorphism induced by $g$, that is, $\tilde{g}(a, \phi)=\left(g(a), \phi \circ g^{-1}\right)$. It is straightforward to check the identity

$$
\tilde{g} \circ \alpha_{f} \circ \tilde{g}^{-1}=\alpha_{(g f)},
$$

where

$$
{ }^{g} f=g \circ f \circ g^{-1}, \quad g \in O(A, q), f \in P(A, q) .
$$

Thus, the autoequivalence crossed module of $\mathscr{C}(A, q)$ is

$$
\mathfrak{A}(\mathscr{C}(A, q)) \simeq(P(A, q), O(A, q))
$$

with structural operations (86) and (87).

5E. Invariants of $\mathfrak{P}(\mathscr{C}(\boldsymbol{A}, \boldsymbol{q}))$. The kernel and the cokernel of the homomorphism $\partial$ are important invariants of a crossed module. Below, we compute the kernel of $\partial$ for the crossed module $\mathfrak{P}(\mathscr{C}(A, q))$. We also describe the cokernel of $\partial$ for the crossed module $\mathfrak{P}(\mathscr{C}(A, q))$ when $\mathscr{E}_{\text {sym }}(\mathscr{C}(A, q))$ is tannakian.

As before, let $A^{\perp} \subset A$ denote the kernel of $\sigma$. Note that $\mathscr{C}\left(A^{\perp},\left.q\right|_{A^{\perp}}\right)=$ $\mathscr{L}_{\text {sym }}(\mathscr{C}(A, q))$ is a symmetric fusion category.

Proposition 5.17. The group homomorphism (61)

$$
j: \operatorname{Pic}\left(\mathscr{C}\left(A^{\perp},\left.q\right|_{A^{\perp}}\right)\right) \rightarrow \operatorname{ker}(\partial)
$$

is an isomorphism.

Proof. The homomorphism $j$ can be explicitly described as follows. For $g$ in $P\left(A^{\perp},\left.q\right|_{A^{\perp}}\right)$ the image $j(g) \in P(A, q)$ is the composition

$$
j(g): \hat{A} \rightarrow \widehat{A^{\perp}} \stackrel{g}{\rightarrow} A^{\perp} \hookrightarrow A,
$$

where the first arrow is the restriction of a character and the last arrow is the embedding.

We will construct the inverse homomorphisms

$$
i: \operatorname{Ker}(\partial) \rightarrow \operatorname{Pic}\left(\mathscr{C}\left(A^{\perp},\left.q\right|_{A^{\perp}}\right)\right)
$$


to $j$. Let $f \in \operatorname{Ker}(\partial)$. Then $f \circ \tilde{\sigma}=0$; that is, $\left.f\right|_{\widehat{A / A^{\perp}}}=0$. By Remark 5.13 we also have $f^{*} \in \operatorname{Ker}(\partial)$, and hence $f^{*} \circ \tilde{\sigma}=0$. Taking the dual we get $\tilde{\sigma} \circ f=0$, that is, $f(\hat{A}) \subset A^{\perp}$. Hence $f$ descends to a homomorphism

$$
i(f): \widehat{A^{\perp}} \cong \hat{A} /\left(\widehat{A / A^{\perp}}\right) \rightarrow A^{\perp},
$$

which is easily seen to be in $P\left(A^{\perp},\left.q\right|_{A^{\perp}}\right)$.

Now let $\mathscr{C}(A, q))$ be a pointed category whose symmetric center $\mathscr{L}_{\text {sym }}(\mathscr{C}(A, q))$ is tannakian. In other words let $\left.q\right|_{A^{\perp}}=1$. Note that in this case the form $q$ descends to $A / A^{\perp}$ (we denote the descendent form by $\tilde{q}$ ). Below, we describe the kernel of the homomorphism (62) for $\mathscr{C}(A, q)$.

Proposition 5.18. Let $\left.q\right|_{A^{\perp}}=1$. Then the kernel of the canonical homomorphism (62)

$$
\operatorname{coker}\left(\operatorname{Pic}(\mathscr{C}(A, q)) \stackrel{\partial}{\rightarrow} \operatorname{Aut}^{\mathrm{br}}(\mathscr{C}(A, q))\right) \rightarrow \operatorname{Aut}\left(A^{\perp}\right)
$$

is isomorphic to the abelian group $\operatorname{Hom}\left(A / A^{\perp}, A^{\perp}\right)$. In other words, the cokernel $C=\operatorname{coker}\left(\operatorname{Pic}(\mathscr{C}(A, q)) \stackrel{\partial}{\rightarrow} \operatorname{Aut}^{\mathrm{br}}(\mathscr{C}(A, q))\right)$ fits into an exact sequence

$$
0 \longrightarrow \operatorname{Hom}\left(A / A^{\perp}, A^{\perp}\right) \longrightarrow C \longrightarrow \operatorname{Aut}\left(A^{\perp}\right) .
$$

Proof. From the commutativity of the diagram

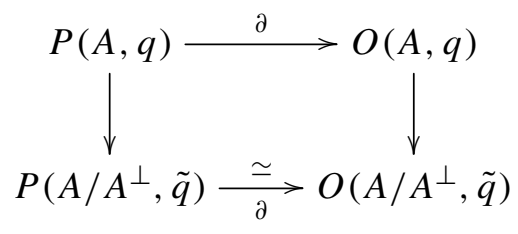

it follows that $\operatorname{coker}(P(A, q) \stackrel{\partial}{\rightarrow} O(A, q))$ coincides with

$$
\operatorname{ker}\left(O(A, q) \rightarrow O\left(A / A^{\perp}, \tilde{q}\right)\right) / \operatorname{im}(\partial) \cap \operatorname{ker}\left(O(A, q) \rightarrow O\left(A / A^{\perp}, \tilde{q}\right)\right) .
$$

Now $\operatorname{ker}\left(O(A, q) \rightarrow O\left(A / A^{\perp}, \tilde{q}\right)\right)$ consists of automorphisms of the form $\mathrm{id}_{A}+\phi$ for $\phi \in \operatorname{Hom}\left(A, A^{\perp}\right)$. Indeed any element of $\operatorname{ker}\left(O(A, q) \rightarrow O\left(A / A^{\perp}, \tilde{q}\right)\right)$ must have this form and conversely any automorphisms of this form preserves $q$ :

$$
q(a+\phi(a))=q(a) q(\phi(a)) \sigma(a, \phi(a))=q(a) .
$$

Note that composition of automorphisms induces the following group operation on $\operatorname{Hom}\left(A, A^{\perp}\right)$ :

$$
\phi * \psi=\phi+\psi+\phi \circ \psi .
$$

It is straightforward that $C=\left\{\phi \in \operatorname{Hom}\left(A, A^{\perp}\right) \mid \mathrm{id}_{A}+\phi\right.$ is invertible $\}$ with the group operation $*$ fits into an exact sequence (88). 
All we need to show now is that the intersection of $\operatorname{im}(\partial)$ with the kernel of $O(A, q) \rightarrow O\left(A / A^{\perp}, \tilde{q}\right)$ is trivial. Assume that $\partial(f)=\mathrm{id}_{A}+\phi$ for $\phi$ in $\operatorname{Hom}\left(A, A^{\perp}\right)$. Then $\phi=-f \circ \tilde{\sigma}$ so that $\operatorname{im}(f) \subset A^{\perp}$. We also have $\partial\left(f^{*}\right)=\operatorname{id}_{A}+\psi$ for $\psi \in \operatorname{Hom}\left(A, A^{\perp}\right)$, which implies that $\operatorname{im}\left(f^{*}\right) \subset A^{\perp}$. Then

$$
\phi=-f \circ \tilde{\sigma}=-\left(\tilde{\sigma} \circ f^{*}\right)^{*}=0 .
$$

\section{Acknowledgments}

We are deeply grateful to V. Drinfeld. The statements of Theorems 3.10 and 4.3 are due to him. We also thank J. Cuadra, V. Ostrik and the anonymous referee for valuable comments.

\section{References}

[Bakalov and Kirillov 2001] B. Bakalov and A. Kirillov, Jr., Lectures on tensor categories and modular functors, University Lecture Series 21, American Mathematical Society, Providence, RI, 2001. MR 2002d:18003 Zbl 0965.18002

[Böckenhauer et al. 2001] J. Böckenhauer, D. E. Evans, and Y. Kawahigashi, "Longo-Rehren subfactors arising from $\alpha$-induction”, Publ. Res. Inst. Math. Sci. 37:1 (2001), 1-35. MR 2002d:46053 Zbl 1090.46047

[Carnovale 2006] G. Carnovale, "The Brauer group of modified supergroup algebras", J. Algebra 305:2 (2006), 993-1036. MR 2008d:16055 Zbl 1135.16043

[Davydov et al. 2011] A. Davydov, L. Kong, and I. Runkel, "Invertible defects and isomorphisms of rational CFTs", Adv. Theor. Math. Phys. 15:1 (2011), 43-69. MR 2888007 Zbl 1246.81319

[Davydov et al. 2013] A. Davydov, D. Nikshych, and V. Ostrik, "On the structure of the Witt group of braided fusion categories", Selecta Math. (N.S.) 19:1 (2013), 237-269. MR 3022755

[Deligne 2002] P. Deligne, “Catégories tensorielles”, Mosc. Math. J. 2:2 (2002), 227-248. MR 2003k:18010 Zbl 1005.18009

[Drinfeld et al. 2010] V. Drinfeld, S. Gelaki, D. Nikshych, and V. Ostrik, "On braided fusion categories, I", Selecta Math. (N.S.) 16:1 (2010), 1-119. MR 2011e:18015 Zbl 1201.18005

[Eilenberg and Mac Lane 1953] S. Eilenberg and S. Mac Lane, "On the groups $H(\Pi, n)$, I", Ann. of Math. (2) 58 (1953), 55-106. MR 15,54b Zbl 0050.39304

[Eilenberg and Mac Lane 1954] S. Eilenberg and S. Mac Lane, "On the groups $H(\Pi, n)$, II: Methods of computation”, Ann. of Math. (2) 60 (1954), 49-139. MR 16,391a Zbl 0055.41704

[Etingof and Ostrik 2004] P. Etingof and V. Ostrik, "Finite tensor categories", Mosc. Math. J. 4:3 (2004), 627-654. MR 2005j:18006 Zbl 1077.18005

[Etingof et al. 2010] P. Etingof, D. Nikshych, and V. Ostrik, "Fusion categories and homotopy theory", Quantum Topol. 1:3 (2010), 209-273. MR 2011h:18007 Zbl 1214.18007

[Goff et al. 2007] C. Goff, G. Mason, and S.-H. Ng, "On the gauge equivalence of twisted quantum doubles of elementary abelian and extra-special 2-groups", J. Algebra 312:2 (2007), 849-875. MR 2008d:16057 Zbl 1171.16021

[Janelidze and Kelly 2001] G. Janelidze and G. M. Kelly, "A note on actions of a monoidal category", Theory Appl. Categ. 9 (2001), 61-91. MR 2003f:18007 Zbl 1009.18005 
[Joyal and Street 1993] A. Joyal and R. Street, "Braided tensor categories", Adv. Math. 102:1 (1993), 20-78. MR 94m:18008 Zbl 0817.18007

[Müger 2003] M. Müger, "On the structure of modular categories", Proc. London Math. Soc. (3) 87:2 (2003), 291-308. MR 2004g:18009 Zbl 1037.18005

[Naidu 2007] D. Naidu, "Categorical Morita equivalence for group-theoretical categories", Comm. Algebra 35:11 (2007), 3544-3565. MR 2008j:18007 Zbl 1143.18009

[Ostrik 2003a] V. Ostrik, "Module categories over the Drinfeld double of a finite group", Int. Math. Res. Not. 2003:27 (2003), 1507-1520. MR 2004h:18005 Zbl 1044.18005

[Ostrik 2003b] V. Ostrik, "Module categories, weak Hopf algebras and modular invariants", Transform. Groups 8:2 (2003), 177-206. MR 2004h:18006 Zbl 1044.18004

[Quillen 1973] D. Quillen, "Higher algebraic $K$-theory, I", pp. 85-147 in Algebraic $K$-theory, I: Higher K-theories (Seattle, WA, 1972), edited by H. Bass, Lecture Notes in Math. 341, Springer, Berlin, 1973. MR 49 \#2895 Zbl 0292.18004

[Van Oystaeyen and Zhang 1998] F. Van Oystaeyen and Y. Zhang, "The Brauer group of a braided monoidal category”, J. Algebra 202:1 (1998), 96-128. MR 99c:18006 Zbl 0909.18005

Communicated by Susan Montgomery

Received 2012-02-06 Revised 2012-11-08 Accepted 2012-11-20

alexei1davydov@gmail.com Department of Mathematics, Ohio University, Athens, $\mathrm{OH}$ 45701, United States

nikshych@math.unh.edu Department of Mathematics, University of New Hampshire, Durham, NH 03824, United States 


\section{Algebra \& Number Theory}

msp.org/ant

\section{EDITORS}

MANAGING EDITOR

Bjorn Poonen

Massachusetts Institute of Technology

Cambridge, USA

\author{
EDITORIAL BOARD CHAIR \\ David Eisenbud \\ University of California \\ Berkeley, USA
}

\section{BOARD OF EDITORS}

Georgia Benkart

Dave Benson

Richard E. Borcherds

John H. Coates

J-L. Colliot-Thélène

Brian D. Conrad

Hélène Esnault

Hubert Flenner

Edward Frenkel

Andrew Granville

Joseph Gubeladze

Ehud Hrushovski

Craig Huneke

Mikhail Kapranov

Yujiro Kawamata

János Kollár

Yuri Manin

Barry Mazur

Philippe Michel
University of Wisconsin, Madison, USA

University of Aberdeen, Scotland

University of California, Berkeley, USA

University of Cambridge, UK

CNRS, Université Paris-Sud, France

University of Michigan, USA

Freie Universität Berlin, Germany

Ruhr-Universität, Germany

University of California, Berkeley, USA

Université de Montréal, Canada

San Francisco State University, USA

Hebrew University, Israel

University of Virginia, USA

Yale University, USA

University of Tokyo, Japan

Princeton University, USA

Northwestern University, USA

Harvard University, USA

École Polytechnique Fédérale de Lausanne
Susan Montgomery

Shigefumi Mori

Raman Parimala

Jonathan Pila

Victor Reiner

Karl Rubin

Peter Sarnak

Joseph H. Silverman

Michael Singer

Vasudevan Srinivas

J. Toby Stafford

Bernd Sturmfels

Richard Taylor

Ravi Vakil

Michel van den Bergh

Marie-France Vignéras

Kei-Ichi Watanabe

Efim Zelmanov
University of Southern California, USA

RIMS, Kyoto University, Japan

Emory University, USA

University of Oxford, UK

University of Minnesota, USA

University of California, Irvine, USA

Princeton University, USA

Brown University, USA

North Carolina State University, USA

Tata Inst. of Fund. Research, India

University of Michigan, USA

University of California, Berkeley, USA

Harvard University, USA

Stanford University, USA

Hasselt University, Belgium

Université Paris VII, France

Nihon University, Japan

University of California, San Diego, USA

\section{PRODUCTION}

production@msp.org

Silvio Levy, Scientific Editor

See inside back cover or msp.org/ant for submission instructions.

The subscription price for 2013 is US \$200/year for the electronic version, and \$350/year ( $\$ 40$, if shipping outside the US) for print and electronic. Subscriptions, requests for back issues and changes of subscribers address should be sent to MSP.

Algebra \& Number Theory (ISSN 1944-7833 electronic, 1937-0652 printed) at Mathematical Sciences Publishers, 798 Evans Hall \#3840, c/o University of California, Berkeley, CA 94720-3840 is published continuously online. Periodical rate postage paid at Berkeley, CA 94704, and additional mailing offices.

ANT peer review and production are managed by EditFLOW ${ }^{\circledR}$ from Mathematical Sciences Publishers.

\section{PUBLISHED BY}

- mathematical sciences publishers

nonprofit scientific publishing

http://msp.org/

(C) 2013 Mathematical Sciences Publishers 


\section{Algebra \& Number Theory}

Volume $7 \quad$ No. $6 \quad 2013$

On the discrete logarithm problem in elliptic curves II

Claus Diem

Identifying Frobenius elements in Galois groups

1325

TIM DOKCHITSER and VLADIMIR DOKCHITSER

Weak approximation for cubic hypersurfaces of large dimension

1353

MIKE SWARBRICK JONES

The Picard crossed module of a braided tensor category

1365

ALEXEI DAVYDOV and DMITRI NIKSHYCH

A Gross-Zagier formula for quaternion algebras over totally real fields

1405

EYAL Z. GOREN and KRISTIN E. LAUTER

Counting rational points over number fields on a singular cubic surface

CHRISTOPHER FREI

On the ample cone of a rational surface with an anticanonical cycle

ROBERT FRIEDMAN

Commuting involutions of Lie algebras, commuting varieties, and simple Jordan algebras

DMITRI I. PANYUSHEV 